九州大学学術情報リポジトリ

Kyushu University Institutional Repository

\title{
A hybrid LES/RANS approach using an anisotropy- resolving algebraic turbulence model
}

$A B E$, Ken-ichi

Department of Aeronautics and Astronautics, Faculty of Engineering, Kyushu University :

Professor

http://hdl. handle. net/2324/4150686

出版情報: International Journal of Heat and Fluid Flow. 26 (2)，pp.204-222，2005-04. Elsevier バージョン：

権利関係 : 


\title{
A hybrid LES/RANS approach using an anisotropy-resolving algebraic turbulence model
}

\author{
K. $\mathrm{Abe}^{1}$ \\ ${ }^{1}$ Department of Aeronautics and Astronautics, Kyushu University, Fukuoka, Japan
}

(Corresponding author: Dr. Ken-ichi Abe, Department of Aeronautics and Astronautics, Kyushu University, Hakozaki, Higashi-ku, Fukuoka 812-8581, Japan, TEL: +81-92-642-3723, FAX: +81-92-642-3752, E-mail: abe@aero.kyushu-u.ac.jp.)

\begin{abstract}
In order to derive a possible path for developing a large eddy simulation (LES) applicable to high Reynolds-number turbulent flows, a hybrid approach connecting LES with the Reynoldsaveraged Navier-Stokes (RANS) modeling in the near-wall region is studied. In contrast to most of the previous studies that have employed linear eddy-viscosity models, in this study, an advanced non-linear eddy-viscosity model is introduced to resolve the near-wall stress anisotropy more correctly. To investigate the model performance in detail, the proposed model is applied to fully-developed plane channel flows with various grid resolutions and at various Reynolds numbers. The grid resolution in the streamwise $(x)$ and spanwise $(z)$ directions ranges from $10^{1}$ to $10^{3}$ in wall unit $\left(\Delta x^{+}\right.$or $\left.\Delta z^{+}\right)$, while $y_{\text {wall }}^{+} \sim 1$ in the wall-normal (y) direction. The present model provides encouraging results for further development of this kind of hybrid LES/RANS model.
\end{abstract}

\section{Keywords}

Large Eddy Simulation, Detached Eddy Simulation, Hybrid LES/RANS Model

Non-Linear Eddy-Viscosity Model, Near-Wall Anisotropy 


\section{Introduction}

Large eddy simulation (LES) is well known as a promising way to predict complex turbulent flows. Although LES needs far fewer grid nodes compared to direct numerical simulation (DNS), there still remains a serious difficulty in its application to very high Reynolds-number $(R e)$ flows. For instance, reasonable LES for a channel flow needs a grid resolution such as $\Delta x^{+} \sim 100$ ( $x$ : the streamwise direction) and $\Delta z^{+} \sim 20$ ( $z$ : the spanwise direction) in the near-wall region, as well as $\Delta y^{+} \sim 1$ in the wall-normal direction, where the no-slip condition is specified at the wall surface. Note that ()$^{+}$denotes a value normalized by the friction velocity $u_{\tau}$. When LES is applied to the channel flow at $R e_{\tau}=10^{4}$, where $\operatorname{Re}_{\tau}\left(=u_{\tau} H / \nu\right)$ is the Reynolds number based on the half channel height $(H)$ and the friction velocity, the aforementioned grid resolution $\Delta z^{+}=20$ means $\Delta z=2 \times 10^{-3} H$ (i.e., 500 grid points per $H$ in $z$-direction), which is unrealistic for a practical LES. Such being the case, the requirement of the grid resolution for high Re LES is significant, even if it is applied to attached wall-shear flows.

To overcome this difficulty, a great deal of effort has gone into the development and improvement of the LES model. The key factor is how we can reduce the computational cost in the near-wall region for very high Re turbulent flows. One promising approach may be, what is called, the "zonal approach." The zonal approach is originally based on the concept of a hybrid approach connecting LES with Reynolds-averaged Navier-Stokes (RANS) modeling in the near-wall region. So far, two representative methods have been studied by a number of research groups. One is known as the "two-layer model" (TLM) and the other is the "detached eddy simulation" (DES). Among TLMs, a representative study was reported by Balaras et al. (1996), where LES is applied in a core region, while another set of equations is separately solved on a finer grid embedded between the edge of the core region and the wall surface. In the DES procedure, on the other hand, a single grid system is adopted to calculate the flow field, where the turbulence model changes from RANS in the near-wall region to LES in the core region. Significant studies were made by Nikitin et al. (2000) and Piomelli et al. (2003), where the fundamental features and problems to be resolved in DES were discussed in detail. Another example was reported by Davidson and Peng (2003), where a two equation turbulence model was adopted in the near-wall region. Temmerman et al. (2003) and Hadziabdic et al. (2003) investigated several kinds of hybrid approach, 
where they discussed the dynamics in the switching region between LES and RANS. Every approach has provided encouraging results and useful knowledge for the development of such a hybrid approach.

On the other hand, very few studies have been reported regarding detailed discussion on the near-wall anisotropy of turbulence when hybrid LES/RANS models are applied to flow predictions. The main reason is that most of the previous LES/RANS approaches have adopted linear eddy-viscosity models (LEVMs), which can never predict the near-wall anisotropy correctly. However, this issue is becoming more important because the RANS model always covers the near-wall region in a hybrid LES/RANS approach. This is, naturally, of particular concern in relation to scalar (heat and mass) transfer at walls, in which the turbulence property of the near-wall layer plays a critical role. When an LEVM is used in the near-wall region, any advanced scalar-transfer model based on the generalized gradientdiffusion hypothesis (Daly and Harlow, 1970) or its higher-order extension (Suga and Abe, 2000; Abe and Suga, 2001) can never be applied because it needs the correct near-wall limiting behavior of the Reynolds-stress anisotropy, in particular the correct wall-normal turbulence.

Another important concern is the influence of the grid resolution on predictive performance. This issue is crucial when the model is applied to the "danger zone" (Nikitin et al., 2000), where the grid resolution is around $0.2 H$ or coarser. It has been said that LES with a coarse grid resolution tends to give very low mean friction coefficient and also to result in an almost one-component turbulence. Recent studies (e.g., Nikitin et al., 2000; Piomelli et al., 2003) have discussed model performance only with grid resolutions of $\Delta z \sim 0.1 H$ (or finer), and few detailed investigations have been reported on model performance with much coarser grid resolutions being used.

The present paper is a contribution to the ongoing search for a better hybrid LES/RANS approach applicable to complex high Re flows. The emphasis of the contribution is on the introduction of an advanced non-linear eddy-viscosity model (NLEVM) to resolve the nearwall stress anisotropy more correctly. To discuss the aforementioned issues, a suitable test case with a very simple geometry is needed because a case with complex geometry is likely to hide the fundamental feature of the RANS model used in the near-wall region. Hence, in this study, the proposed model is carefully applied to fully-developed plane channel flows with various grid resolutions and at various Reynolds numbers. By processing the computational results, the present study discusses the model performance in detail. 


\section{Turbulence Models}

\subsection{Governing equations}

The filtered (or Reynolds-averaged) governing equations for an incompressible turbulent flow may be written as

$$
\begin{gathered}
\frac{\partial \bar{U}_{i}}{\partial x_{i}}=0 \\
\frac{\mathrm{D} \bar{U}_{i}}{\mathrm{D} t}=-\frac{1}{\rho} \frac{\partial \bar{P}}{\partial x_{i}}+\frac{\partial}{\partial x_{j}}\left\{\nu\left(\frac{\partial \bar{U}_{i}}{\partial x_{j}}+\frac{\partial \bar{U}_{j}}{\partial x_{i}}\right)-\tau_{i j}\right\}
\end{gathered}
$$

where $\overline{(~)}$ denotes a filtered value in the LES region or a Reynolds-averaged value in the RANS region, respectively. In Eq. (2), $\rho, \bar{P}, \bar{U}_{i}$ and $\nu$ respectively denote density, filtered static pressure, filtered velocity and kinematic viscosity. The sub-grid scale (SGS) stress $\tau_{i j}$ is originally expressed as

$$
\tau_{i j}=\overline{U_{i} U_{j}}-\bar{U}_{i} \bar{U}_{j}
$$

Note that $\tau_{i j}$ coincides with the following general expression for the Reynolds-stress tensor in the RANS region:

$$
\tau_{i j}=\overline{u_{i} u_{j}}
$$

where $u_{i}$ is defined as $u_{i}=U_{i}-\bar{U}_{i}$.

\subsection{NLEVM in the RANS region}

In order to improve the prediction accuracy in the near-wall region, an anisotropy-resolving algebraic turbulence model is introduced. The following is the summary of the model (Abe et al., 2003) used in the present study, which is categorized in the two-equation NLEVM.

The Reynolds stress $\overline{u_{i} u_{j}}$ in the RANS region may be evaluated as follows:

$$
\begin{aligned}
\overline{u_{i} u_{j}} & =\frac{2}{3} k \delta_{i j}-2 k \tau C_{B}\left[1+\left\{1-f_{w}(26)\right\} f_{s 1}\right] S_{i j} \\
& +4 k \tau^{2} C_{D} C_{B}\left\{1-f_{w}(26)\right\}\left\{-\left(S_{i k} \Omega_{k j}-\Omega_{i k} S_{k j}\right)+\left(1-f_{s 2}\right)\left(S_{i k} S_{k j}-\frac{\delta_{i j}}{3} S^{2}\right)\right\} \\
& +2 k^{w} b_{i j}
\end{aligned}
$$

where $k\left(=\overline{u_{i} u_{i}} / 2\right)$ is the turbulence energy. In Eq. (5), $S_{i j}$ and $\Omega_{i j}$ respectively denote the strain-rate tensor and the vorticity tensor as

$$
S_{i j}=\frac{1}{2}\left(\frac{\partial \bar{U}_{i}}{\partial x_{j}}+\frac{\partial \bar{U}_{j}}{\partial x_{i}}\right), \quad \Omega_{i j}=\frac{1}{2}\left(\frac{\partial \bar{U}_{i}}{\partial x_{j}}-\frac{\partial \bar{U}_{j}}{\partial x_{i}}\right)
$$


The model function $f_{w}$ is modeled as follows (Abe et al., 1997):

$$
f_{w}(\xi)=\exp \left\{-\left(\frac{n^{*}}{\xi}\right)^{2}\right\}
$$

where $\xi$ is a prescribed constant. In Eq. $(7), n^{*}\left(=(\nu \varepsilon)^{1 / 4} n / \nu\right)$ is the non-dimensional wall distance with Kolmogorov scale (Abe et al., 1994), where $\varepsilon$ is the dissipation rate of $k$ and $n$ is uniquely determined as the shortest distance from all the wall surfaces. In the model, the characteristic time scale $\tau$ and the model coefficients are as follows (Abe et al., 2003):

$$
\begin{gathered}
\tau=\frac{\nu_{t}}{k}, \quad \nu_{t}=C_{\mu} f_{\mu} \frac{k^{2}}{\varepsilon}, \quad C_{B}=\frac{1}{1+\frac{22}{3}\left(C_{D} \tau\right)^{2} \Omega^{2}+\frac{2}{3}\left(C_{D} \tau\right)^{2}\left(\Omega^{2}-S^{2}\right) f_{B}} \\
f_{s 1}=f_{r 1} f_{r 2} C_{s 1}\left(C_{D} \tau\right)^{2}\left(\Omega^{2}-S^{2}\right), \quad f_{s 2}=f_{r 1} f_{r 2}\left\{1+C_{s 2} C_{D} \tau(\Omega-S)\right\} \\
f_{B}=1+C_{\eta} C_{D} \tau(\Omega-S), \quad f_{r 1}=\frac{\Omega^{2}-S^{2}}{\Omega^{2}+S^{2}}, \quad f_{r 2}=\frac{S^{2}}{\Omega^{2}+S^{2}} \\
S^{2}=S_{m n} S_{m n}, \quad \Omega^{2}=\Omega_{m n} \Omega_{m n}, \quad S=\sqrt{S^{2}}, \quad \Omega=\sqrt{\Omega^{2}} \\
C_{D}=0.8, \quad C_{\mu}=0.12, \quad C_{\eta}=100, \quad C_{s 1}=0.15 C_{\eta}, \quad C_{s 2}=0.07 C_{\eta}
\end{gathered}
$$

To represent the damping effects of viscosity, use is made of Abe et al.'s (1997) damping function:

$$
f_{\mu}=\left[1+\frac{35}{R_{t}^{\frac{3}{4}}} \exp \left\{-\left(\frac{R_{t}}{30}\right)^{\frac{3}{4}}\right\}\right]\left\{1-f_{w}(26)\right\}
$$

where $R_{t}\left(=k^{2} / \nu \varepsilon\right)$ is the turbulent Reynolds number.

In Eq. (5), ${ }^{w} b_{i j}$ is introduced to improve the predictive performance of the near-wall stress anisotropy as follows (Abe et al., 2003):

$$
\begin{aligned}
{ }^{w} b_{i j}=f_{w}(26)[ & -\alpha_{w} \frac{1}{2}\left(d_{i} d_{j}-\frac{\delta_{i j}}{3} d_{k} d_{k}\right) \\
& +\left(1-f_{r 1}^{2}\right) \tau_{d}^{2}\left\{-\frac{\beta_{w} C_{w}}{1+C_{w} \tau_{d}^{2} \sqrt{S^{2} \Omega^{2}}}\left(S_{i k} \Omega_{k j}-\Omega_{i k} S_{k j}\right)\right. \\
& \left.\left.\quad+\frac{\gamma_{w} C_{w}}{1+C_{w} \tau_{d}^{2} S^{2}}\left(S_{i k} S_{k j}-\frac{\delta_{i j}}{3} S^{2}\right)\right\}\right]
\end{aligned}
$$

where

$$
d_{i}=\frac{N_{i}}{\sqrt{N_{k} N_{k}}}, \quad N_{i}=\frac{\partial n}{\partial x_{i}}, \quad \tau_{d}=\left\{1-f_{w}(15)\right\} \frac{k}{\varepsilon}+f_{w}(15) \delta_{w} \sqrt{\frac{\nu}{\varepsilon}}
$$


In this study, the following combined model is used to represent the near-wall fragment:

$$
\begin{aligned}
& { }^{w} b_{i j}=f_{\tau}{ }^{w 1} b_{i j}+\left(1-f_{\tau}\right){ }^{w 2} b_{i j}, \quad f_{\tau}=\exp \left[-3\left\{1-f_{w}(26)\right\} \frac{k}{\varepsilon} \sqrt{S^{2}}\right] \\
& { }^{w 1} b_{i j}=\text { Eq. (10) } \operatorname{with}\left(\alpha_{w}=1, \quad \beta_{w}=\frac{1}{4}, \quad \gamma_{w}=1.5, \quad \delta_{w}=1.0, \quad C_{w}=0.5\right) \\
& { }^{w 2} b_{i j}=\text { Eq. (10) } \operatorname{with}\left(\alpha_{w}=0, \quad \beta_{w}=\frac{13}{30}, \quad \gamma_{w}=0.6, \quad \delta_{w}=3.0, \quad C_{w}=1.0\right)
\end{aligned}
$$

Turbulence energy and its dissipation rate are determined from the usual form of the transport equations:

$$
\begin{gathered}
\frac{\mathrm{D} k}{\mathrm{D} t}=\frac{\partial}{\partial x_{j}}\left\{\left(\nu+\frac{\nu_{t}}{\sigma_{k}}\right) \frac{\partial k}{\partial x_{j}}\right\}-\overline{u_{i} u_{j}} \frac{\partial \bar{U}_{i}}{\partial x_{j}}-\varepsilon \\
\frac{\mathrm{D} \varepsilon}{\mathrm{D} t}=\frac{\partial}{\partial x_{j}}\left\{\left(\nu+\frac{\nu_{t}}{\sigma_{\varepsilon}}\right) \frac{\partial \varepsilon}{\partial x_{j}}\right\}-C_{\varepsilon 1} \frac{\varepsilon}{k} \overline{u_{i} u_{j}} \frac{\partial \bar{U}_{i}}{\partial x_{j}}-C_{\varepsilon 2} f_{\varepsilon} \frac{\varepsilon^{2}}{k}
\end{gathered}
$$

where

$$
\begin{gathered}
C_{\varepsilon 1}=1.45, \quad C_{\varepsilon 2}=1.83, \quad \sigma_{k}=\frac{1.2}{f_{t}}, \quad \sigma_{\varepsilon}=\frac{1.5}{f_{t}} \\
f_{t}=1+5.0 f_{w}(5), \quad f_{\varepsilon}=\left[1-0.3 \exp \left\{-\left(\frac{R_{t}}{6.5}\right)^{2}\right\}\right]\left\{1-f_{w}(3.3)\right\}
\end{gathered}
$$

Further detailed description of the model is given in Abe et al. (2003). It is noted that this RANS model provides good predictions for flow fields with massive separations as well as for attached wall-shear flows (Jang et al., 2002; Abe et al., 2003).

\subsection{SGS model in the LES region}

To connect the LES and RANS regions smoothly, it may be desirable to adopt the same fundamental model expressions in both two regions. Thus, as the first attempt, the model expressions in Eqs. (5) - (11) are also adopted for modeling $\tau_{i j}$ in the LES region with some modifications, though the turbulence energy and the dissipation rate are replaced for the following SGS values:

$$
k \rightarrow k_{S}, \quad \varepsilon \rightarrow \varepsilon_{S}
$$

where $k_{S}$ and $\varepsilon_{S}$ are originally defined as

$$
k_{S}=\frac{1}{2}\left(\overline{U_{i} U_{i}}-\bar{U}_{i} \bar{U}_{i}\right), \quad \varepsilon_{S}=\nu\left(\overline{\frac{\partial U_{i}}{\partial x_{j}} \frac{\partial U_{i}}{\partial x_{j}}}-\frac{\partial \bar{U}_{i}}{\partial x_{j}} \frac{\partial \bar{U}_{i}}{\partial x_{j}}\right)
$$


In this study, following Inagaki et al. (2002), $k_{S}$ is modeled based on scale-similarity modeling (Bardina et al., 1980) as follows:

$$
k_{S}=C_{k} \frac{1}{2}\left(\bar{U}_{i}-\widehat{\bar{U}}_{i}\right)^{2}
$$

where $C_{k}$ is the model constant and $C_{k}=4.5$ is adopted in this study (Horiuti, 1993). In Eq. (18), ( ) denotes the filtering operator, for which the Simpson rule is adopted (Inagaki et al., 2002). In modeling $\varepsilon_{S}$, the following model is adopted:

$$
\varepsilon_{S}=\frac{k_{S}^{3 / 2}}{\Delta}+\frac{2 \nu k_{S}}{n^{2}}
$$

where $\Delta$ is the representative SGS width defined as $\left(\Delta_{x} \Delta_{y} \Delta_{z}\right)^{1 / 3}$. Note that the second term in Eq. (19) is introduced to improve the near-wall limiting behavior on the wall surface.

Concerning the time scale $\tau$ in Eq. (8), the following form proposed by Abe and Suga (2001) is adopted:

$$
\tau=C_{S} f_{S} \frac{\Delta}{\sqrt{k_{S}}}, \quad C_{S}=0.12, \quad f_{S}=1-f_{w}(26)
$$

where $f_{S}$ is the model function introduced to ensure the near-wall limiting behavior (Abe and Suga, 2001). Note that the near-wall limiting behavior of $\tau$ in Eq. (20) coincides with that in Eq. (8), i.e. $\tau \propto y^{1}$. As for ${ }^{w} b_{i j}$ in Eq. (10), the following model constants are adopted:

$$
{ }^{w} b_{i j}=\text { Eq. (10) with }\left(\alpha_{w}=0, \quad \beta_{w}=\frac{13}{30}, \quad \gamma_{w}=0.6, \quad \delta_{w}=6.0, \quad C_{w}=1.0\right)
$$

In calculating $\tau_{d}$ in Eq. (11), $k$ and $\varepsilon$ are simply replaced by $k_{S}$ and $\varepsilon_{S}$, respectively.

\subsection{The present hybrid approach}

To determine the switching location between the LES and RANS regions, the present approach introduces the DES concept by Nikitin et al. (2000), though the form used is considerably different. First, $\tau_{i j}$ in Eq. (2) is modeled as follows:

$$
\tau_{i j}=\left(1-f_{h b}\right){\overline{u_{i} u_{j}}}_{(R A N S)}+f_{h b} \tau_{i j(L E S)}
$$

where $f_{h b}$ is the model function to connect the LES and RANS regions smoothly (see for example, Hamba, 2001; Temmerman et al., 2003; Hadziabdic et al., 2003). In Eq. (22), $f_{h b}$ is close to 1 in the region away from the wall, where full LES is adopted. On the other hand, 
the RANS calculation is performed in the near-wall region, where $f_{h b}$ must become close to 0 . In this study, the following expression is adopted for $f_{h b}$ :

$$
f_{h b}=1-\exp \left\{-\left(\frac{n}{C_{h b} \Delta_{\|}}\right)^{6}\right\}
$$

where $\Delta_{\|}$is the length scale representing the region where RANS is applied. Following the previous DES concept by Nikitin et al. (2000), the present $\Delta_{\|}$is determined based on grid resolution. In a sense, $\Delta_{\|}$provides the scale of grid-resolved turbulent structures. Although some options have been proposed to determine $\Delta_{\|}$as

$$
\begin{gathered}
\Delta_{\| 1}=\left(\Delta_{x} \Delta_{y} \Delta_{z}\right)^{1 / 3} \\
\Delta_{\| 2}=\max \left(\Delta_{x}, \Delta_{y}, \Delta_{z}\right) \\
\Delta_{\| 3}=\sqrt{{\Delta_{x}}^{2}+\Delta_{y}{ }^{2}+\Delta_{z}{ }^{2}}
\end{gathered}
$$

(Nikitin et al., 2000; Piomelli et al., 2003), an alternative definition was adopted in this study as

$$
\Delta_{\|}=\sqrt{\frac{\Delta_{x} \Delta_{y} \Delta_{z}}{\min \left(\Delta_{x}, \Delta_{y}, \Delta_{z}\right)}}
$$

Equation (25) can be rewritten as

$$
\Delta_{\|}=\sqrt{\max \left(\Delta_{x} \Delta_{y}, \Delta_{y} \Delta_{z}, \Delta_{z} \Delta_{x}\right)}
$$

Note that in case of $\Delta_{x}=\Delta_{z} \gg \Delta_{y}$ as seen in Nikitin et al. (2000), Eq. (26) gives the same value as $\Delta_{\| 2}$ does. Equation (26) is also recognized as

$$
\Delta_{\|}=\sqrt{\text { the maximum area among the cell faces }}
$$

By using Eq. (27), one can reasonably determine $\Delta_{\|}$even in the non-orthogonal or the unstructured grid system, though some ambiguity may be inevitable due to the grid topology. As for the constant in Eq. (23), the present study adopts $C_{h b}=4.0$. Note that the influence of $C_{h b}$ on the predictive performance will be discussed later.

To connect the LES and RANS regions in calculating the turbulence energy and the dissipation rate, the following procedure was used. In solving Eqs. (13) and (14) numerically, they need to be discretized. A resulting matrix form to be solved in the RANS region may be generally expressed as

$$
\mathbf{A}_{R A N S} \mathbf{Q}=\mathbf{B}_{R A N S}
$$


where $\mathbf{Q}$ denotes $k$ or $\varepsilon$. On the other hand, a similar form in the LES region may be obtained as

$$
\mathbf{A}_{L E S} \mathbf{Q}=\mathbf{B}_{L E S}
$$

In this study, $k$ and $\varepsilon$ in the LES region are algebraically calculated and thus Eq. (29) may be rewritten as

$$
\mathbf{I} \mathbf{Q}=\mathbf{Q}_{L E S}
$$

where $\mathbf{I}$ is the unit matrix and $\mathbf{Q}_{L E S}$ is $k_{S}$ or $\varepsilon_{S}$ calculated by Eq. (18) or (19), respectively. In this study, the two forms in the LES and RANS regions are combined with the model function $f_{h b}$ in Eq. (23) as

$$
\left\{\left(1-f_{h b}\right) \mathbf{A}_{R A N S}+f_{h b} \mathbf{I}\right\} \mathbf{Q}=\left(1-f_{h b}\right) \mathbf{B}_{R A N S}+f_{h b} \mathbf{Q}_{L E S}
$$

\section{Test Cases and Computational Conditions}

To investigate the characteristics of the present hybrid approach, the present model is applied to fully-developed plane channel flows with various grid resolutions and at various Reynolds numbers. The computational parameters are summarized in Table 1. The Reynolds number $\left(R e_{\tau}=u_{\tau} H / \nu\right)$ ranges from 180 to 10000 . The lower $R e$ cases $\left(R e_{\tau}=180,395\right)$ are selected for detailed discussion on basic model performance. On the other hand, the higher $R e$ cases $\left(R e_{\tau}=1000,3000,10000\right)$ provide useful knowledge of the model performance when the model is applied to very high Re flows.

Another important concern is the effect of grid resolution on model performance. To study this issue, four grid resolutions are selected as shown in Table 1 . Note that $\Delta_{\|}$in the table denotes the value in the near-wall region, where $\Delta y$ is much smaller than both $\Delta x$ and $\Delta z$. The last letter of the codes in the table (i.e., "A", "B", "C" or "D") identifies the grid resolution in $x$ - $z$ plane. For instance, "C1E3A" means the calculation at $R e_{\tau}=1000$ with grid resolution of $\Delta_{\|}=0.1$ in the $x-z$ plane. The grid resolution in cases with the last letter "A" $\left(\Delta_{\|}=0.1\right)$ is usually recognized as relatively coarse for LES, although it may still be applicable to very low Re cases. On the other hand, the grid resolution in the cases with "B" $\left(\Delta_{\|}=0.2\right)$ is thought to be about the start of the "danger zone." The cases with "C" $\left(\Delta_{\|}=0.4\right)$ have a much coarser grid resolution which is no longer applicable to LES. As mentioned before, LES with such a coarse grid resolution tends to give very low mean friction 
coefficient and result in almost one-component turbulence. To avoid this crucial problem, one option may be such that the RANS model fully covers the whole boundary layer when the grid resolution becomes "dangerous." In this study, this important issue will be discussed with the computational results. Finally, the cases with "D" $\left(\Delta_{\|}=0.05\right)$ have been computed with yet finer grids over the same domain size as the cases with "A." These are introduced to investigate the trend of the model performance as the grid resolution becomes finer.

Calculations were performed with the finite-volume procedure STREAM of Lien and Leschziner (1994a), followed by several improvements and substantially upgraded by Apsley and Leschziner (1999). This method uses collocated storage on a grid. The second-order central difference scheme was used for the discretization of each term, except for the convection terms of $k$ (Eq. (13)) and $\varepsilon$ (Eq. (14)) which were discretized by the UMIST scheme (Lien and Leschziner, 1994b), a TVD implementation of the QUICK scheme. The solution algorithm is based on the SIMPLE scheme. As for the time integration, the second-order Crank-Nicolson scheme was employed. It is noted that stable computations were possible for all the present test cases.

\section{Results and Discussion}

\subsection{Basic performance with no hybrid approach}

First, to investigate the basic performance of the present model, the flow calculations were performed with only the RANS or the SGS model used for the whole computational domain. Figure 1 gives the full RANS results for the mean velocity, turbulence energy, Reynolds shear stress and Reynolds normal stresses at $R e_{\tau}=395$, by comparison to the DNS data (Moser et al., 1999). As seen in the figure, fairly good agreement is achieved, including the correct behavior of the near-wall anisotropy. In particular, the model returns the correct near-wall limiting behavior of the wall-normal turbulence.

Figure 2 compares the full LES results at $R e_{\tau}=180$. In this calculation, the grid resolution is the same as that in case $\operatorname{C180D}\left(\Delta_{\|}=0.05\right)$. The present results show good agreement with the DNS data (Moser et al., 1999). This grid resolution is well known to be enough for LES at such a low Reynolds number (Abe and Suga, 2001). As seen in the figure, the modeled stresses are very small in this case and most of the stresses are successfully resolved. From the results, it is understood that the present program code has the capability 
of providing reasonable results when it is applied to LES with a suitable grid resolution.

On the other hand, Fig. 3 shows the results of the full LES at $R e_{\tau}=395$ with the grid resolution of $\Delta_{\|}=0.1$. In contrast to the last case in Fig. 2, the predictions give considerable errors. Typical features of LES with a coarse grid resolution are seen in Fig. 3. First, the mean-velocity profile shows a considerable shift up from the DNS data. This also means serious underestimation in values of the skin friction coefficient. As seen in Fig. 3 (b), the Reynolds shear stress is underestimated, which corresponds to the shift up in the mean-velocity profile. It is also found that the turbulence energy is overestimated in the near-wall region. As seen in Fig. 3 (d), the overestimation in the turbulence energy is caused by an overestimation in the streamwise turbulence, while the other two components (i.e. the wall-normal and the spanwise) are considerably underestimated. An insufficient grid resolution tends to suppress the energy redistribution from the streamwise to the other two components, and then the streamwise turbulence is overpredicted and the flow tends to be of one-component turbulence. This suppression of the energy redistribution causes a serious decrease in the production of the resolved shear stress, leading to the aforementioned underprediction in Fig. 3 (b).

From Fig. 3, it may be concluded that there is some further room for improving the calibration in the SGS-model expressions used here. However, it should be noted that (full) LES also depends strongly on the computational scheme, i.e. the order of discretization (second-order, fourth-order or higher) and the discretization method (regular, collocation or staggered), as well as the grid resolution. On the contrary, as seen in Fig. 1, the performance of the RANS model in the near-wall region usually depends only on the resolution in the wall-normal direction. In this sense, it is expected that the hybrid LES/RANS model may reduce problems of this kind.

\subsection{Performance of the present hybrid approach}

Figure 4 shows the distribution of the present switching function $f_{h b}$ with $C_{h b}=4.0$. As described before, one important issue in the hybrid LES/RANS model is how we can minimize the decrease in accuracy when it is applied to the "danger zone." Therefore in this study, as a first attempt, $f_{h b}$ is modeled so that the present hybrid model can return to an almost wholly RANS calculation when it is applied to such a coarse grid as seems insufficient for LES. 
Figure 5 compares instantaneous velocity-vector plots in $y$ - $z$ plane at $R e_{\tau}=395$ for three grid resolutions, i.e. $\Delta_{\|}=0.05,0.1$ and 0.2 . In the figure, the region between the two lines, i.e. $f_{h b}=0.1$ and 0.9 , represents the switching region. Near-wall vortex structures are clearly identified in Fig. 5 (a), though the vortex structures become larger and weaker as the grid resolution becomes coarser (Figs. 5 (b) and (c)). As will be shown below, turbulence properties evaluated by the RANS model become larger and more dominant in such coarser grid cases.

Figures $6-9$ give the results at $R e_{\tau}=395$ with four grid resolutions, i.e. $\Delta_{\|}=0.05$, 0.1, 0.2 and 0.4, respectively. As seen in Figs. 6 and 7, the predicted profiles of the mean velocity, turbulence energy and Reynolds-shear stress are smooth and acceptable for finer grid resolutions (i.e. C395D and C395A), though the total (resolved+modeled) predicted turbulence energy is slightly overpredicted in the near-wall region. Reasonable prediction of the mean velocity also provides a good prediction of the skin friction coefficient. Concerning the Reynolds normal stresses, all the components are generally predicted well, though some overestimations are seen for streamwise turbulence. It is noted that in contrast to the results in Fig. 3, the resolved streamwise turbulence is reduced and the total (resolved+modeled) level is reasonable. Although good performance is obtained in the near-wall (RANS) region, some suppression of the energy redistribution from the streamwise to the wall-normal and spanwise components is seen in the switching region, resulting in a little stronger stressanisotropy prediction. From the figures, it is found that such a suppression of the energy redistribution occurs at around the location of the maximum resolved Reynolds-shear stress.

Figure 8 provides the results when the model is applied to the "danger zone" (C395B). Although the results are still encouraging, some up-shift is seen in the mean-velocity profile. It is thought that the decrease in accuracy is caused by the inaccurate anisotropy prediction of turbulence in the LES region, as seen in Fig. 8 (d). Due to the coarse grid resolution, the lower energy redistribution is clearer than that in Fig. 7, resulting in a stronger stress anisotropy, not only in the switching region at around $y / H \sim 0.6$, but also in the whole LES region. It is of interest that the location of $y / H \sim 0.6$ is also where the maximum resolved Reynolds-shear stress occurs (see Fig. 8 (b)). As is mentioned before, the suppression of the energy redistribution causes a decrease in the resolved shear stress. To correct this defect, the mean velocity provides a larger gradient, leading to an up-shift in the mean velocity, as seen in Fig. 8 (a). This defect is thought to be similar to what was seen in previous studies 
(Nikitin et al., 2000; Piomelli et al., 2003). In this respect, there still remains some margin for improvement. Fortunately, however, the up-shift in the mean velocity is limited to within $10 \%$, therefore the predictions are still practically useful.

On the other hand, Fig. 9 shows the results when the model is applied to a much coarser grid resolution which is usually inapplicable to LES (C395C). As described above, the present switching function $f_{h b}$ is modeled so that the present hybrid model can return to almost full RANS calculation in the case of such a coarse grid resolution (see Fig. 4). As is expected, the present model provides almost full RANS results similar to those in Fig. 1. Even if a much coarser grid resolution is employed, the present model always gives the full RANS results. It means that the present model can cover every grid resolution to within an error of $10 \%$, at least at this Reynolds number.

From the present results, it is understood that the present hybrid LES/RANS model generally provides reasonable predictions for both the mean velocity and the total (resolved + modeled) turbulent properties in a wide range of grid resolutions. It is also notable that for all the test cases, the total wall-normal turbulence $\overline{v v}$ is reasonably well predicted with the correct near-wall limiting behavior, owing to the introduction of a wall-anisotropy resolving NLEVM such as the present model (Abe et al., 2003). It is also shown that the present switching function $f_{h b}$ in Eq. (23) generally connects the LES and RANS regions smoothly, though a stronger stress anisotropy is seen in the switching region even with finer grid resolutions (cases C395D and C395A). It is also noted that LES tends to have a considerable effect on the computation even in the region where $f_{h b}$ has just a small non-zero value $\left(f_{h b} \sim 0.1\right.$ or less $)$, e.g. the location $y / H \sim 0.5$ in case C395B.

To compare the performance between LEVM and NLEVM, Fig. 10 shows the results with an LEVM adopted. In this calculation, only the linear term (the first line) in Eq. (5) was used for modeling $\tau_{i j}$ in both the LES and RANS regions. It is found from the figure that the predictive performance is generally acceptable for the mean velocity, turbulence energy and Reynolds shear stress, though some overpredictions are seen in the mean-velocity and turbulence-energy profiles. As for the Reynolds normal stresses, however, it is clearly seen that inaccurate predictions are obtained. This is the natural consequence of the introduction of an LEVM which can never reproduce the stress anisotropy correctly. 


\subsection{Effect of the switching location}

To investigate the effect of the switching location on the predictive performance, a calculation was performed with a different constant selected for $C_{h b}$ in Eq. (23). Figure 11 shows the distribution of $f_{h b}$ with $C_{h b}=1.0$.

Figure 12 shows the results for $C_{h b}=1.0$ at $R e_{\tau}=395$ with the same grid as in case C395C $\left(\Delta_{\|}=0.4\right)$. As found from the comparison between Figs. 11 and 4 , the $f_{h b}$ profile with $C_{h b}=1.0$ and $\Delta_{\|}=0.4$ is similar to that with $C_{h b}=4.0$ and $\Delta_{\|}=0.1$. In this sense, the comparison between Figs. 12 and 7 gives us a very important insight. It is clearly seen that the velocity profile in Fig. 12 has a considerable increase from the DNS data in the LES region. In the near-wall region, however, the prediction is generally reasonable not only for the mean velocity but also for turbulent properties, including stress anisotropy, though some overestimation is seen for the turbulence energy and the streamwise turbulence.

As seen in Fig. 12, it is thought that the decrease in accuracy is due to the inaccurate prediction of turbulence in the LES region, which is similar to that shown in Fig. 8. An important feature is more clearly seen from a comparison between the stress distributions in Figs. 12 and 7. The coarser grid resolution provides a much stronger stress anisotropy in the switching region at around $y / H \sim 0.4$, which indicates an insufficient energy redistribution. This causes a serious decrease in the production of the resolved shear stress, and the mean velocity tends to give a larger gradient as a result, leading to a considerable up-shift in the mean velocity. As seen in Fig. 12 (b), the resolved shear stress in the switching region is much lower than that in Fig. 7 (b).

To remedy this problem, various strategies have been proposed. Piomelli et al. (2003) have suggested an artificial backscatter model. The backscatter model enhances the redistribution of turbulence and thus it may have the potential to improve the prediction accuracy in Fig. 12. However, considering the acceptable results shown in Figs. 6 - 9, the present study has concluded that the use of $f_{h b}$ with $C_{h b}=4.0$ is more preferable in covering all the flow conditions. Although the present model provides reasonable results for higher $R e$ cases as shown below, this issue needs further discussion in the future. 


\subsection{Higher Reynolds-number cases}

Figures 13 - 15 give the calculated results for higher $R e$ cases $\left(R e_{\tau}=1000,3000,10000\right)$ with the present hybrid model $\left(C_{h b}=4.0\right)$. As is seen from the figures, the present model provides encouraging results for both the mean velocity and the Reynolds shear stress, though slight differences are still seen among the velocity profiles with different grid resolutions at the same Reynolds number. In all cases, the mean-velocity profiles are connected smoothly between the LES and RANS regions. It is also understood that the profiles of the total (resolved + modeled) Reynolds shear stress are reasonable and show no conflict with known results. As for the turbulence energy, some differences are still seen among profiles with different grid resolutions. However, the maximum value in each total (resolved + modeled) $k^{+}$shows a reasonable level even for cases at $R e_{\tau}=10000$. Since several research groups have obtained results for the total turbulence energy that are excessively overestimated in the near-wall region, the present results are thought to be promising.

The error in the prediction of the skin friction coefficient is now investigated. Following Piomelli et al. (2003), the error is defined as

$$
\text { Error }=\frac{C_{f}-C_{f, \text { Dean }}}{C_{f, \text { Dean }}} \times 100
$$

where $C_{f}$ is

$$
C_{f}=\frac{\tau_{w}}{\rho U_{b}^{2} / 2}
$$

In Eq. (33), $\tau_{w}\left(=\rho \nu \partial \bar{U} /\left.\partial y\right|_{w}\right)$ and $U_{b}$ denote the mean wall stress and the bulk mean velocity, respectively. The reference wall stress $C_{f, \text { Dean }}$ is evaluated by

$$
C_{f, \text { Dean }}=0.073 R e_{b}{ }^{-1 / 4}
$$

(Dean, 1978), with $R e_{b}=2 U_{b} H / \nu$. Note that in the cases of $R e_{\tau}=180$ and $395, C_{f, \text { Dean }}$ is replaced with $C_{f, D N S}$ as evaluated from the DNS data by Moser et al. (1999). The present error for all the test cases is summarized in Table 2 and Fig. 16. As found from both the table and the figure, the present model provides reasonable predictions of $C_{f}$ to within an error of $12 \%$.

As seen in Fig. 16, the present model tends to give too high values for $C_{f}$ in high $R e$ cases. However, it should be noted that the error for high Re cases is on the positive side, in contrast to the fact that most of the previous studies (Nikitin et al., 2000; Piomelli et al., 2003) have obtained low predictions for $C_{f}$. The present results are completely different from 
what is seen in the previous studies and also seen in Fig. 12. In fact, this high prediction is mainly caused by the properties of the RANS model used in this study. It should be noted again that similar predictions of mean velocity can be obtained at high Reynolds numbers up to $R e_{\tau}=10^{4}\left(R e_{b} \sim 5 \times 10^{5}\right)$ in a wide range of grid resolutions (see Figs. $13-15$ ). Therefore, the present results are very encouraging for further development of LES/RANS modeling of this kind.

\section{Concluding Remarks}

A hybrid approach connecting LES with the RANS modeling in the near-wall region was studied. In contrast to most of the previous studies that employed linear eddy-viscosity models, in this study an advanced non-linear eddy-viscosity model was introduced to resolve the near-wall stress anisotropy more correctly. The present model was applied to fullydeveloped plane channel flows with various grid resolutions and at various Reynolds numbers. The main conclusions derived from this study are as follows:

- The introduction of an anisotropy-resolving NLEVM is very effective in improving the accuracy of the total (resolved+modeled) Reynolds stresses predicted in the nearwall region. In particular, the total wall-normal turbulence $\overline{v v}$ is reasonably predicted with the correct near-wall limiting behavior regardless of the grid resolution. Stable computations for all the test cases may indicate the suitability of the present model to more complex turbulent flows.

- The present switching function (Eq. (23)) connects the LES and RANS regions smoothly. Comparison with the DNS data shows that the predicted profiles of the mean velocity, turbulence energy and Reynolds-shear stress are smooth and acceptable. Regarding the Reynolds normal stresses, all components are generally predicted well. However, some suppression of the energy redistribution from the streamwise to the wall-normal and the spanwise components is seen in the switching region, resulting in a stronger stressanisotropy prediction. In this study, such a suppression of the energy redistribution occurs at around the location of the maximum resolved Reynolds-shear stress.

- The predicted mean-velocity profiles give reasonable predictions of the skin friction coefficient within an error of $12 \%$ for the present test cases at Reynolds numbers up to $R e_{\tau}=10^{4}\left(R e_{b} \sim 5 \times 10^{5}\right)$. Even if the present model is applied to the "danger zone," 
where the grid resolution is around $0.2 \mathrm{H}$ or coarser, it still gives reasonable predictions because it returns to an almost wholly RANS calculation.

Finally, some future areas of study are discussed below. Although the present results are encouraging for further development of this kind of hybrid LES/RANS model, there remain several areas to be improved. The switching location from RANS to LES in this study may be a little too far from the wall surface compared to previous DESs (Nikitin et al., 2000; Piomelli et al., 2003). As found from the present results, however, this choice is necessary at this stage to cover every grid resolution from an engineering point of view (see for example, Hadziabdic et al., 2003). In this sense, the performance of the SGS model used in the LES region is very important because it has a crucial impact on the determination of the switching location. It is expected that improvement of the "LES" performance with coarse grid resolutions, especially in the switching region, will result in a switching location closer to the wall surface.

On the other hand, the present definition for $\Delta_{\|}$in the switching function (Eq. (23)) is based on the grid resolution mainly in the streamwise and spanwise directions. In this respect, further investigations may be needed to introduce some physics-oriented factors into

the switching algorithm (see for example, Batten et al., 2004), which would lead to the improvement of the total predictive performance for complex high Re turbulent flows with impingement and separation.

\section{Acknowledgements}

This research was partially supported by Grant-in-Aids for Scientific Research, No.15360450, No.15106013, sponsored by the Ministry of Education, Culture, Sports, Science and Technology, Japan. The author wishes to express his appreciation to Professor M.A. Leschziner of Imperial College of Science, Technology and Medicine (IC), London, UK for the support in using the STREAM code. The author also wishes to give his thanks to Mr. L. Temmerman of IC for his valuable discussion on this topic. 


\section{References}

Abe, K., Kondoh T., and Nagano Y. (1994). A New Turbulence Model for Predicting Fluid Flow and Heat Transfer in Separating and Reattaching Flows - I. Flow Field Calculations. Int. J. Heat Mass Transfer, 37, 139-151.

Abe, K., Kondoh, T., and Nagano, Y. (1997). On Reynolds stress expressions and near-wall scaling parameters for predicting wall and homogeneous turbulent shear flows. Int. J. Heat Fluid Flow, 18, 266-282.

Abe, K., Jang, Y.J., and Leschziner, M.A. (2003). An investigation of wall-anisotropy expressions and length-scale equations for non-linear eddy-viscosity models. Int. J. Heat Fluid Flow, 24, 181-198.

Abe, K., and Suga, K. (2001). Towards the development of a Reynolds-averaged algebraic turbulent scalar-flux model. Int. J. Heat Fluid Flow, 22, 19-29.

Apsley, D.D. and Leschziner, M.A. (1999). Advanced turbulence modelling of separated flow in a diffuser. Flow, Turbulence and Combustion, 63, 81-112.

Batten, P., Goldberg, U., and Chakravarthy, S. (2004). Interfacing statistical turbulence closures with large-eddy simulation. AIAA J., 42, 485-492.

Balaras, E., Benocci, C., and Piomelli, U. (1996). Two-layer approximate boundary conditions for large-eddy simulations. AIAA J., 34, 1111-1119.

Bardina, J., Ferziger, J.H., and Reynolds, W.C. (1980). Improved subgrid scale models for large eddy simulation. AIAA Paper, 80-1357.

Daly, B.J., and Harlow, F.H. (1970). Transport equations in turbulence. Phys. Fluids, 13, 2634-2649.

Davidson, L., and Peng, S.H. (2003). Hybrid LES-RANS modelling: a one-equation SGS model combined with a $k$ - $\omega$ model for predicting recirculating flows. Int. J. Numer. Meth. Fluids, 43, 1003-1018.

Dean, R.B. (1978). Reynolds number dependence of skin friction and other bulk flow variables in two-dimensional rectangular duct flow. J. Fluids Eng., 100, 215-223.

Hanjalic, K, Hadziabdic, M., Temmerman, L., and Leschziner, M.A. (2004). Merging LES and RANS strategies: zonal or seamless coupling? Direct and Large Eddy Simu- 
lation V (R. Friedrich et al. (eds)), Kluwer Academic Publ., 451-464.

Hamba, F. (2001). An attempt to combine large eddy simulation with the $k-\varepsilon$ model in a channel-flow calculation. Theoret. Comput. Fluid Dynamics, 14, 323-336.

Horiuti, K. (1993). A proper velocity scale for modeling subgrid-scale eddy viscosities in large eddy simulation. Phys. Fluids A, 5, 146-157.

Inagaki, M., Kondoh, T., and Nagano, Y. (2002). A mixed-time-scale SGS model with fixed model-parameters for practical LES. In: Engineering Turbulence Modelling and Experiments - 5, edited by W. Rodi and N. Fueyo, Mallorca, 257-266.

Jang, Y.-J., Leschziner, M.A., Abe, K., and Temmerman, L. (2002). Investigation of AnisotropyResolving Turbulence Models by Reference to Highly-Resolved LES Data for Separated Flow. Flow, Turbulence and Combustion, 69, 161-203.

Lien, F.S., and Leschziner, M.A. (1994a). A general non-orthogonal collocated finite volume algorithm for turbulent flow at all speeds incorporating second-moment turbulencetransport closure, Part 1: Computational implementation. Comput. Methods Appl. Mech. Engrg, 114, 123-148.

Lien, F.S., and Leschziner, M.A. (1994b). Upstream monotonic interpolation for scalar transport with application to complex turbulent flows. Int. J. Num. Meths. In Fluids, 19, $527-548$.

Moser, R.D., Kim, J., and Mansour, N.N. (1999). Direct numerical simulation of turbulent channel flow up to $R e_{\tau}=590$. Phys. Fluids, 11, 943-945.

Nikitin, N.V., Nicoud, F., Wasistho, B., Squires, K.D., and Spalart, P.R. (2000). An approach to wall modeling in large-eddy simulations. Phys. Fluids, 12, 1629-1632.

Piomelli, U., Balaras, E., Pasinato, H., Squaires, K.D., and Spalart, P.R. (2003). The inner-outer layer interface in large-eddy simulations with wall-layer models. Int. J. Heat and Fluid Flow, 24, 538-550.

Suga, K., and Abe, K. (2000). Nonlinear eddy viscosity modeling for turbulence and heat transfer near wall and shear-free boundaries. Int. J. Heat Fluid Flow, 21, 37-48.

Temmerman, L., Leschziner, M.A., and Hanjalic, K. (2003). A combined RANS-LES strategy with arbitrary interface location for near-wall flows. Proc. 3rd Symposium on Turbulence and Shear Flow Phenomena, Sendai, June, 929-934. 
Table 1: Computational parameters.

\begin{tabular}{lcccccccccc}
\hline \hline Case & $R e_{\tau}$ & Grid numbers & Domain $(x-z)$ & $\Delta x$ & $\Delta z$ & $\Delta_{\|}$ & $\Delta y_{c}$ & $\Delta_{\|}{ }^{+}$ & $y_{w}{ }^{+}$ & $\Delta t$ \\
\hline C180A & 180 & $31 \times 61 \times 31$ & $6 \mathrm{H} \times 1.5 \mathrm{H}$ & 0.2 & 0.05 & 0.1 & 0.09 & 18 & 0.18 & $5 \times 10^{-3}$ \\
$\mathrm{C} 395 \mathrm{~A}$ & 395 & $31 \times 61 \times 31$ & $6 \mathrm{H} \times 1.5 \mathrm{H}$ & 0.2 & 0.05 & 0.1 & 0.09 & 40 & 0.4 & $5 \times 10^{-3}$ \\
$\mathrm{C} 1 \mathrm{E} 3 \mathrm{~A}$ & 1000 & $31 \times 61 \times 31$ & $6 \mathrm{H} \times 1.5 \mathrm{H}$ & 0.2 & 0.05 & 0.1 & 0.11 & 100 & 0.3 & $4 \times 10^{-3}$ \\
$\mathrm{C} 3 \mathrm{E} 3 \mathrm{~A}$ & 3000 & $31 \times 61 \times 31$ & $6 \mathrm{H} \times 1.5 \mathrm{H}$ & 0.2 & 0.05 & 0.1 & 0.11 & 300 & 0.9 & $4 \times 10^{-3}$ \\
$\mathrm{C} 1 \mathrm{E} 4 \mathrm{~A}$ & 10000 & $31 \times 61 \times 31$ & $6 \mathrm{H} \times 1.5 \mathrm{H}$ & 0.2 & 0.05 & 0.1 & 0.14 & 1000 & 1.0 & $3 \times 10^{-3}$ \\
$\mathrm{C} 180 \mathrm{~B}$ & 180 & $31 \times 61 \times 31$ & $12 \mathrm{H} \times 3 \mathrm{H}$ & 0.4 & 0.1 & 0.2 & 0.09 & 36 & 0.18 & $5 \times 10^{-3}$ \\
$\mathrm{C} 395 \mathrm{~B}$ & 395 & $31 \times 61 \times 31$ & $12 \mathrm{H} \times 3 \mathrm{H}$ & 0.4 & 0.1 & 0.2 & 0.09 & 79 & 0.4 & $5 \times 10^{-3}$ \\
$\mathrm{C} 1 \mathrm{E} 3 \mathrm{~B}$ & 1000 & $31 \times 61 \times 31$ & $12 \mathrm{H} \times 3 \mathrm{H}$ & 0.4 & 0.1 & 0.2 & 0.11 & 200 & 0.3 & $4 \times 10^{-3}$ \\
$\mathrm{C} 3 \mathrm{E} 3 \mathrm{~B}$ & 3000 & $31 \times 61 \times 31$ & $12 \mathrm{H} \times 3 \mathrm{H}$ & 0.4 & 0.1 & 0.2 & 0.11 & 600 & 0.9 & $4 \times 10^{-3}$ \\
$\mathrm{C} 1 \mathrm{E} 4 \mathrm{~B}$ & 10000 & $31 \times 61 \times 31$ & $12 \mathrm{H} \times 3 \mathrm{H}$ & 0.4 & 0.1 & 0.2 & 0.14 & 2000 & 1.0 & $3 \times 10^{-3}$ \\
$\mathrm{C} 180 \mathrm{C}$ & 180 & $31 \times 61 \times 31$ & $24 \mathrm{H} \times 6 \mathrm{H}$ & 0.8 & 0.2 & 0.4 & 0.09 & 72 & 0.18 & $5 \times 10^{-3}$ \\
$\mathrm{C} 395 \mathrm{C}$ & 395 & $31 \times 61 \times 31$ & $24 \mathrm{H} \times 6 \mathrm{H}$ & 0.8 & 0.2 & 0.4 & 0.09 & 158 & 0.4 & $5 \times 10^{-3}$ \\
$\mathrm{C} 1 \mathrm{E} 3 \mathrm{C}$ & 1000 & $31 \times 61 \times 31$ & $24 \mathrm{H} \times 6 \mathrm{H}$ & 0.8 & 0.2 & 0.4 & 0.11 & 400 & 0.3 & $4 \times 10^{-3}$ \\
$\mathrm{C} 3 \mathrm{E} 3 \mathrm{C}$ & 3000 & $31 \times 61 \times 31$ & $24 \mathrm{H} \times 6 \mathrm{H}$ & 0.8 & 0.2 & 0.4 & 0.11 & 1200 & 0.9 & $4 \times 10^{-3}$ \\
$\mathrm{C} 1 \mathrm{E} 4 \mathrm{C}$ & 10000 & $31 \times 61 \times 31$ & $24 \mathrm{H} \times 6 \mathrm{H}$ & 0.8 & 0.2 & 0.4 & 0.14 & 4000 & 1.0 & $3 \times 10^{-3}$ \\
C180D & 180 & $61 \times 61 \times 61$ & $6 \mathrm{H} \times 1.5 \mathrm{H}$ & 0.1 & 0.025 & 0.05 & 0.09 & 9 & 0.18 & $5 \times 10^{-3}$ \\
C395D & 395 & $61 \times 61 \times 61$ & $6 \mathrm{H} \times 1.5 \mathrm{H}$ & 0.1 & 0.025 & 0.05 & 0.09 & 20 & 0.4 & $5 \times 10^{-3}$ \\
$\mathrm{C} 1 \mathrm{E} 3 \mathrm{D}$ & 1000 & $61 \times 61 \times 61$ & $6 \mathrm{H} \times 1.5 \mathrm{H}$ & 0.1 & 0.025 & 0.05 & 0.11 & 50 & 0.3 & $4 \times 10^{-3}$ \\
$\mathrm{C} 3 \mathrm{E} 3 \mathrm{D}$ & 3000 & $61 \times 61 \times 61$ & $6 \mathrm{H} \times 1.5 \mathrm{H}$ & 0.1 & 0.025 & 0.05 & 0.11 & 150 & 0.9 & $4 \times 10^{-3}$ \\
$\mathrm{C} 1 \mathrm{E} 4 \mathrm{D}$ & 10000 & $61 \times 61 \times 61$ & $6 \mathrm{H} \times 1.5 \mathrm{H}$ & 0.1 & 0.025 & 0.05 & 0.14 & 500 & 1.0 & $3 \times 10^{-3}$ \\
\hline \hline
\end{tabular}


Table 2: Percent error in the predictions of the skin friction coefficient $C_{f}$.

\begin{tabular}{lccccc}
\hline \hline Case & C180A & C395A & C1E3A & C3E3A & C1E4A \\
\hline \% Error & -0.4 & -0.9 & -4.1 & 2.0 & 8.9 \\
\hline \hline Case & C180B & C395B & C1E3B & C3E3B & C1E4B \\
\hline \% Error & -1.3 & -7.6 & -7.8 & -1.1 & 4.5 \\
\hline \hline & & & & & \\
\hline \hline Case & C180C & C395C & C1E3C & C3E3C & C1E4C \\
\hline$\%$ Error & -0.5 & -2.0 & -3.6 & -0.8 & 5.8 \\
\hline \hline & & & & & \\
\hline \hline Case & C180D & C395D & C1E3D & C3E3D & C1E4D \\
\hline$\%$ Error & -3.8 & 0.2 & -6.1 & 1.5 & 11.8 \\
\hline \hline
\end{tabular}




\section{Figure captions}

1 Predictions of full RANS $\left(R e_{\tau}=395\right)$ : (a) Mean velocity; (b) Reynolds shear stress; (c) Turbulence energy; (d) Reynolds normal stresses.

2 Predictions of full LES ( $R e_{\tau}=180$, the same grid as C180D): (a) Mean velocity; (b) Reynolds shear stress; (c) Turbulence energy; (d) Reynolds normal stresses.

3 Predictions of full LES $\left(R e_{\tau}=395\right.$, the same grid as C395A): (a) Mean velocity;

(b) Reynolds shear stress; (c) Turbulence energy; (d) Reynolds normal stresses.

4 Distributions of switching function $f_{h b}\left(C_{h b}=4.0\right)$.

$5 \quad$ Instantaneous velocity-vector plots in $y$-z plane $\left(R e_{\tau}=395\right)$ : (a) $\Delta_{\|}=0.05$; (b) $\Delta_{\|}=0.1 ;(\mathrm{c}) \Delta_{\|}=0.2$.

6 Comparison with DNS data $\left(R e_{\tau}=395, \Delta_{\|}=0.05\right)$ : (a) Mean velocity; (b) Reynolds shear stress; (c) Turbulence energy; (d) Reynolds normal stresses.

7 Comparison with DNS data $\left(R e_{\tau}=395, \Delta_{\|}=0.1\right)$ : (a) Mean velocity; (b) Reynolds shear stress; (c) Turbulence energy; (d) Reynolds normal stresses.

8 Comparison with DNS data $\left(R e_{\tau}=395, \Delta_{\|}=0.2\right)$ : (a) Mean velocity; (b) Reynolds shear stress; (c) Turbulence energy; (d) Reynolds normal stresses.

9 Comparison with DNS data $\left(R e_{\tau}=395, \Delta_{\|}=0.4\right.$ ): (a) Mean velocity; (b) Reynolds shear stress; (c) Turbulence energy; (d) Reynolds normal stresses.

10 Computational results for $\operatorname{LEVM}\left(R e_{\tau}=395, \Delta_{\|}=0.1\right)$ : (a) Mean velocity; (b) Reynolds shear stress; (c) Turbulence energy; (d) Reynolds normal stresses.

11 Distributions of switching function $f_{h b}\left(C_{h b}=1.0\right)$.

12 Computational results for $C_{h b}=1.0\left(R e_{\tau}=395, \Delta_{\|}=0.4\right)$ : (a) Mean velocity; (b) Reynolds shear stress; (c) Turbulence energy; (d) Reynolds normal stresses.

13 Comparison of predictions at $R e_{\tau}=1000$ : (a) Mean velocity; (b) Reynolds shear stress; (c) Turbulence energy.

14 Comparison of predictions at $R e_{\tau}=3000$ : (a) Mean velocity; (b) Reynolds shear stress; (c) Turbulence energy.

15 Comparison of predictions at $R e_{\tau}=10000$ : (a) Mean velocity; (b) Reynolds shear stress; (c) Turbulence energy.

16 Percent error in the predictions of the skin friction coefficient $C_{f}$. 


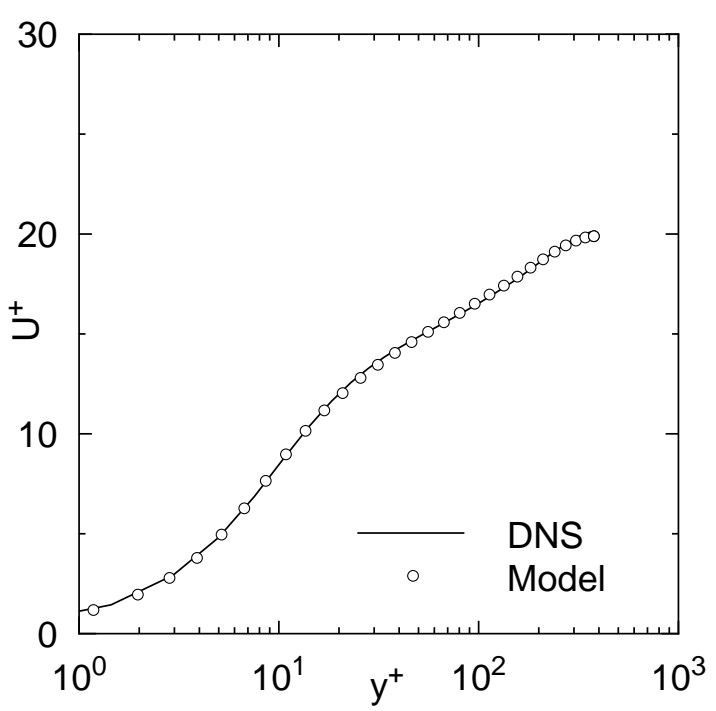

(a)

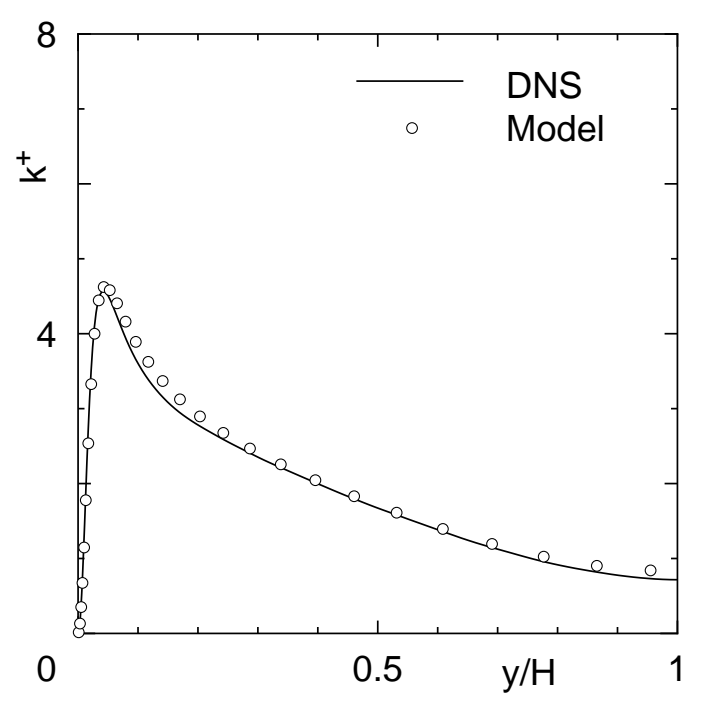

(c)

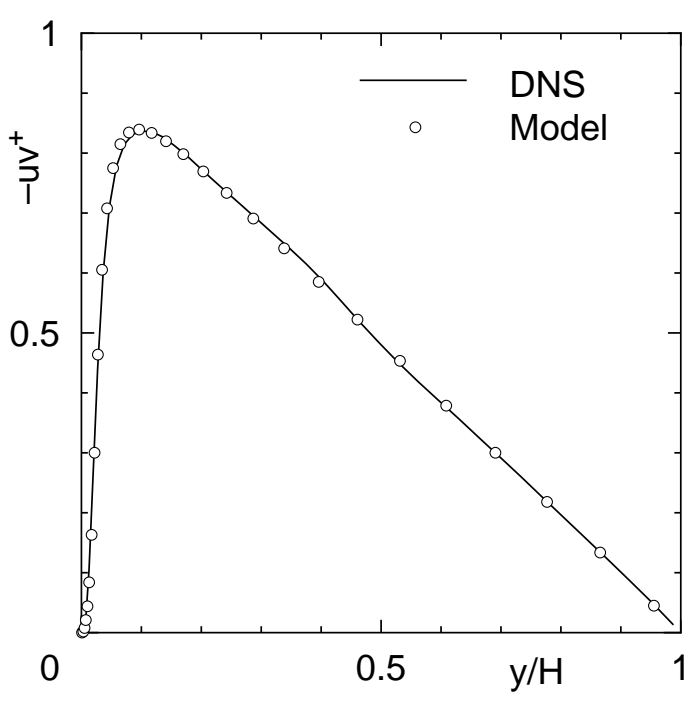

(b)

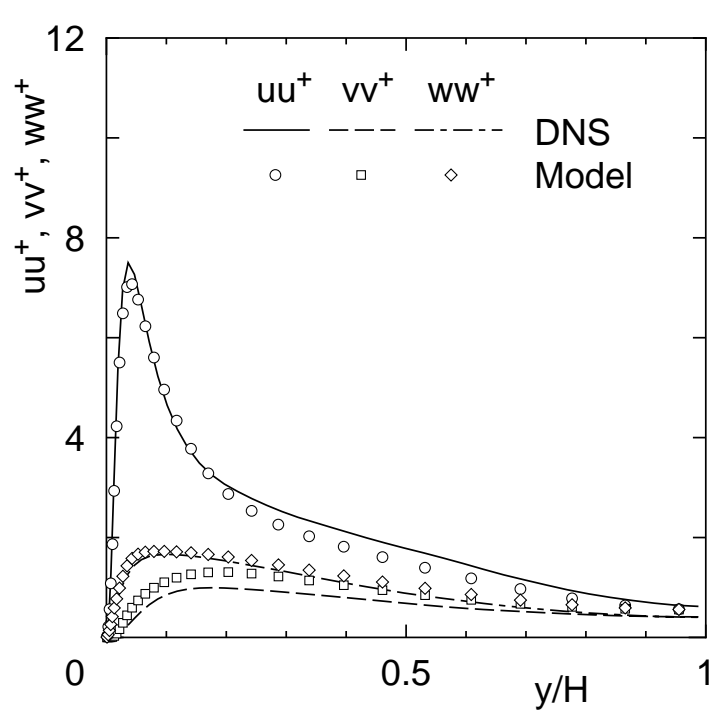

(d)

Figure 1:

$\ll \mathrm{K}$. Abe» 


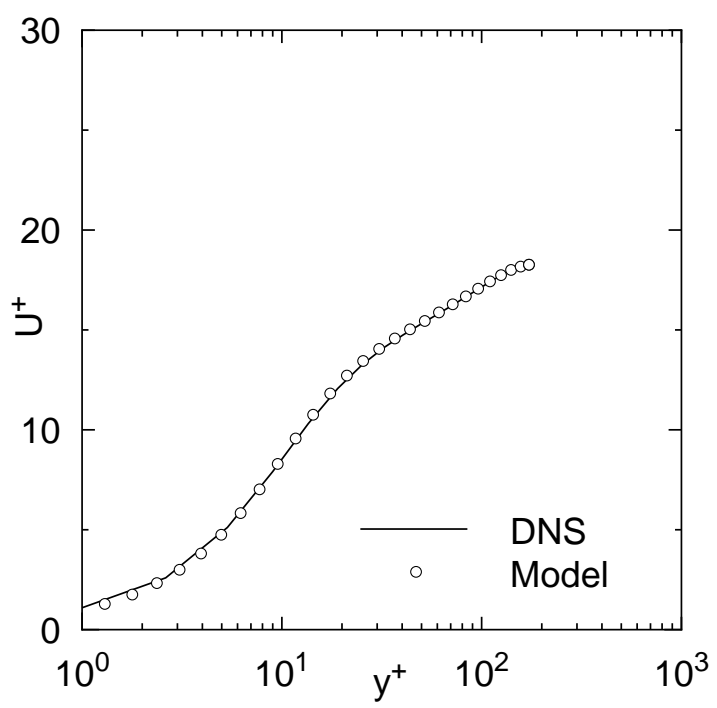

(a)

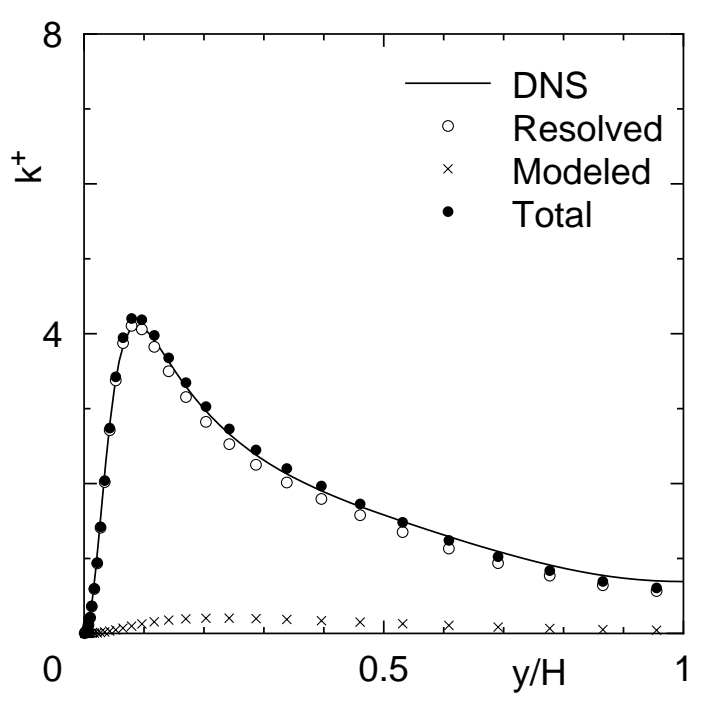

(c)

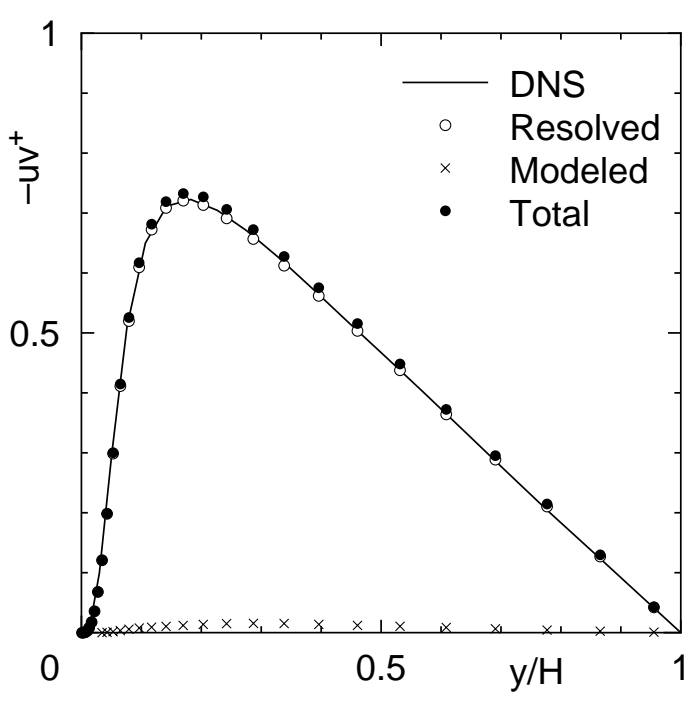

(b)

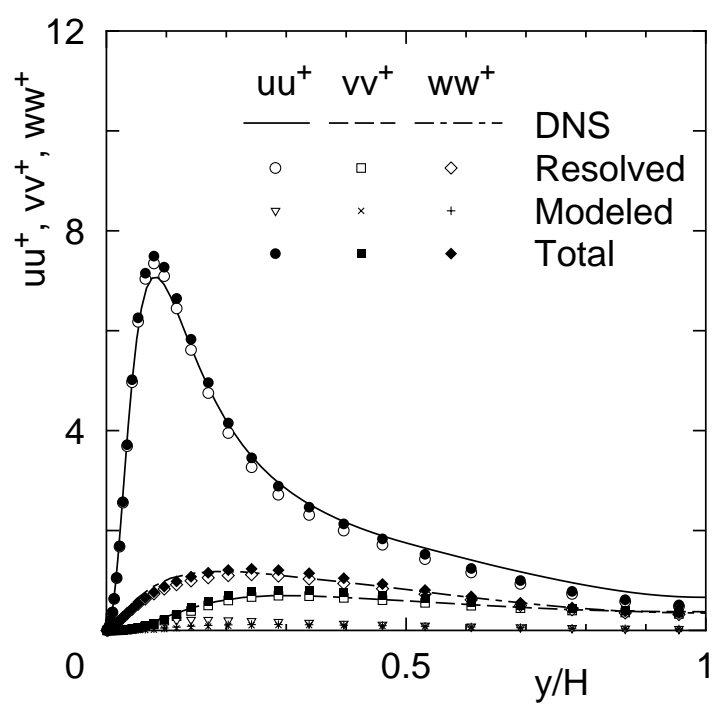

(d)

Figure 2:

$\ll \mathrm{K}$. Abe» 


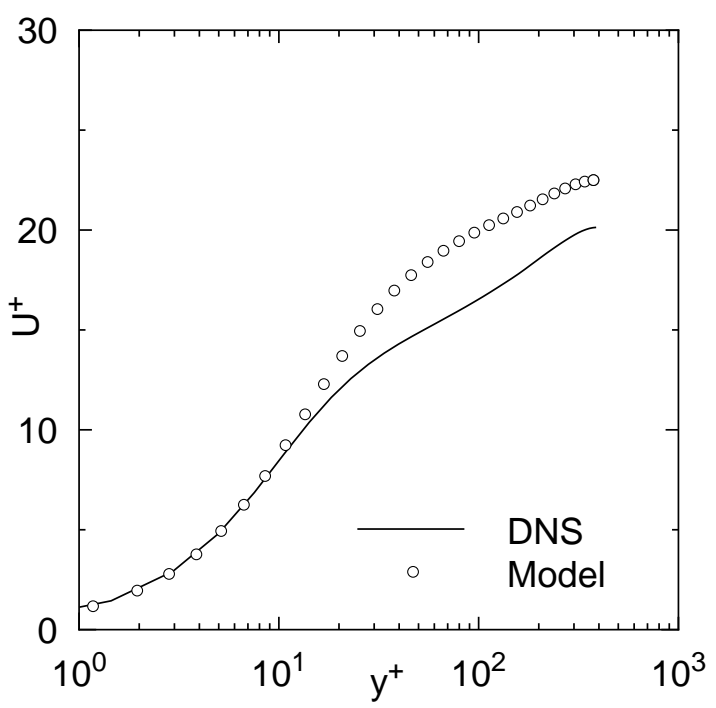

(a)

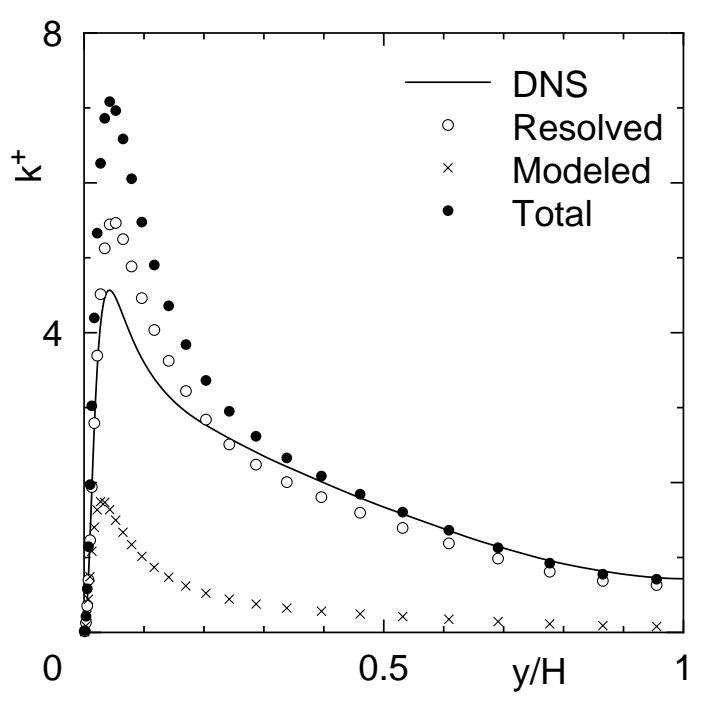

(c)

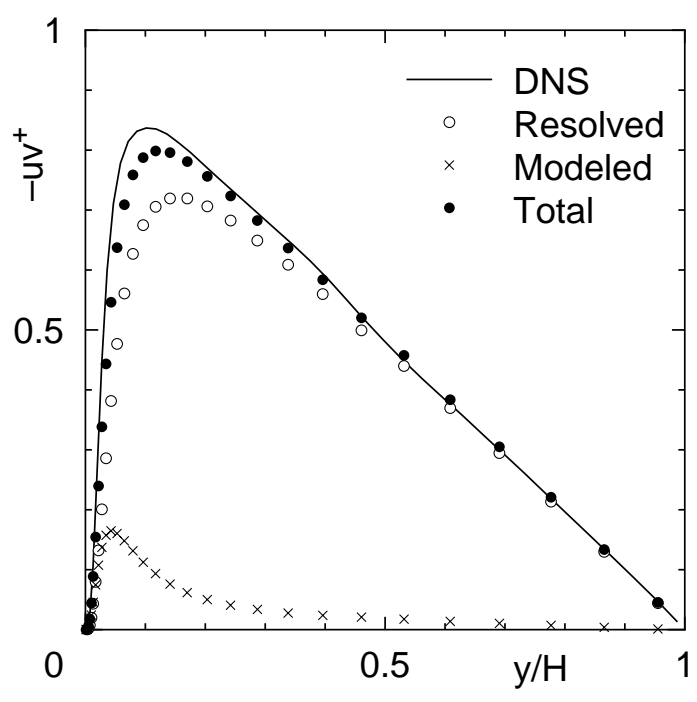

(b)

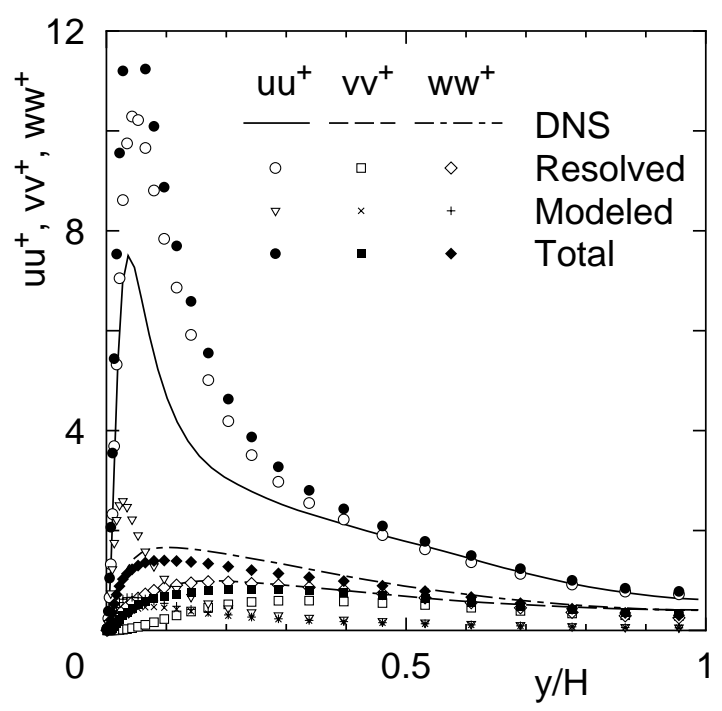

(d)

Figure 3:

$\ll \mathrm{K}$. Abe» 


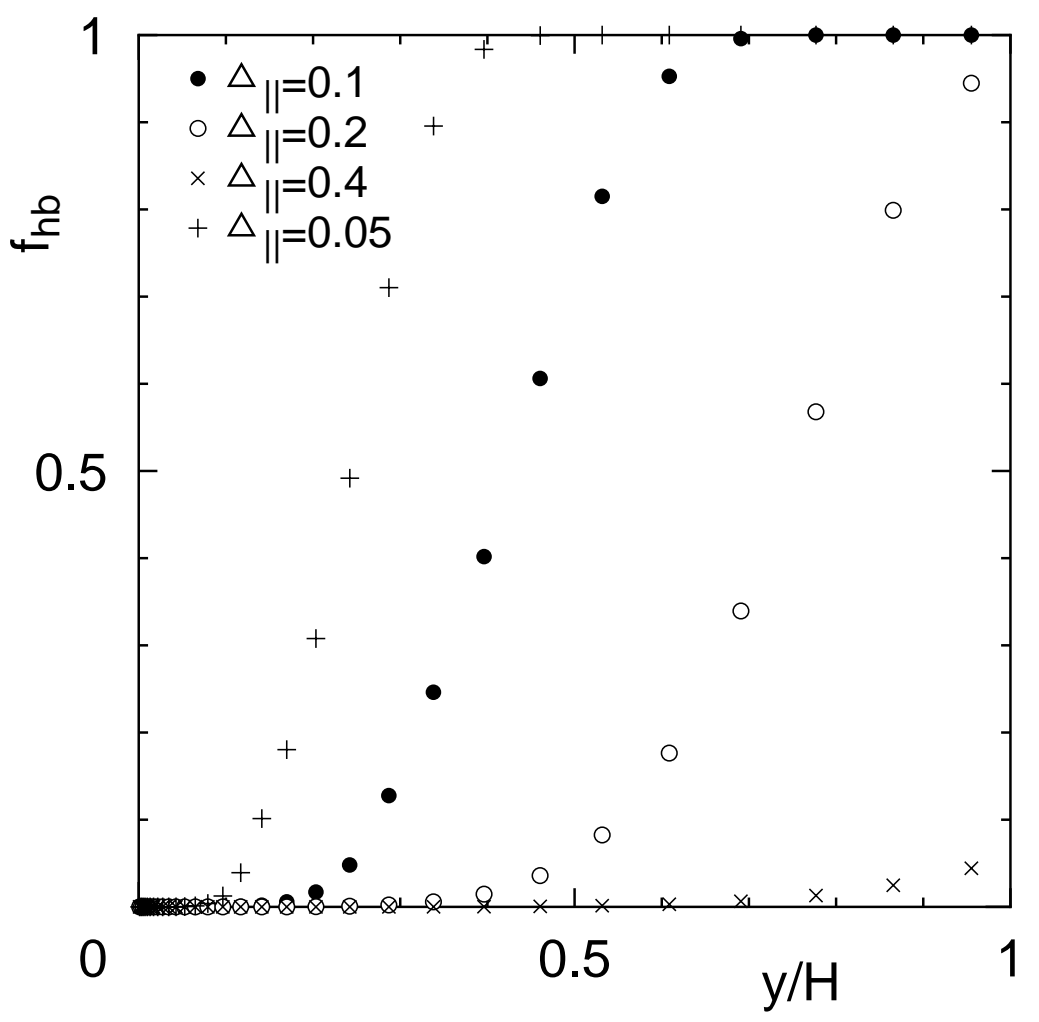

Figure 4: $\quad \ll \mathrm{K}$. Abe» 


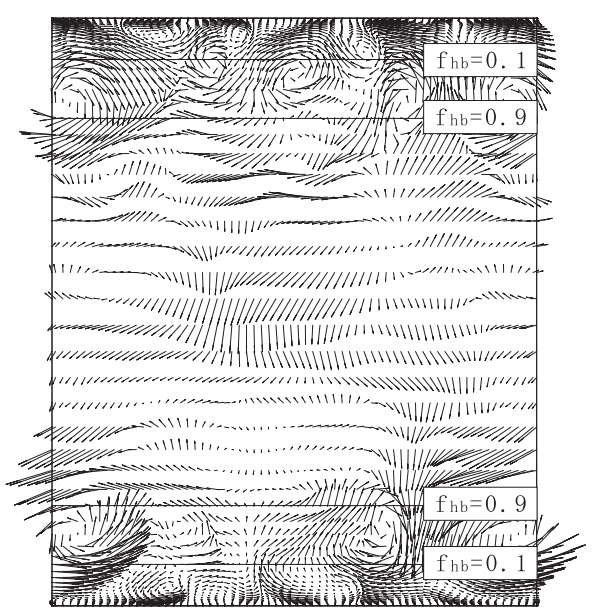

(a)

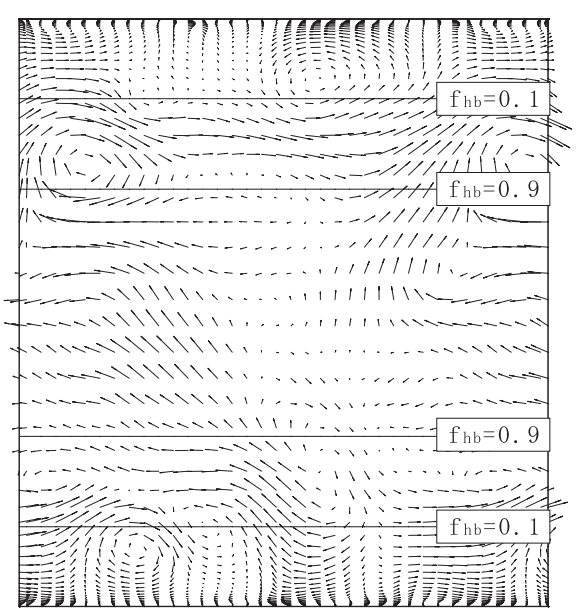

(b)

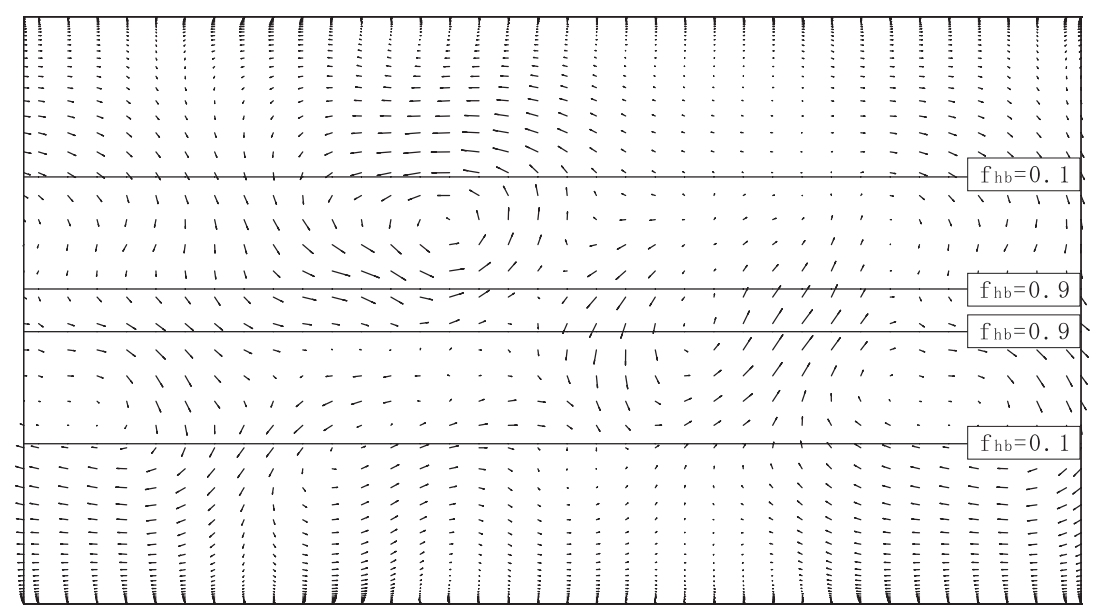

(c)

Figure 5:

$\ll \mathrm{K}$. Abe» 


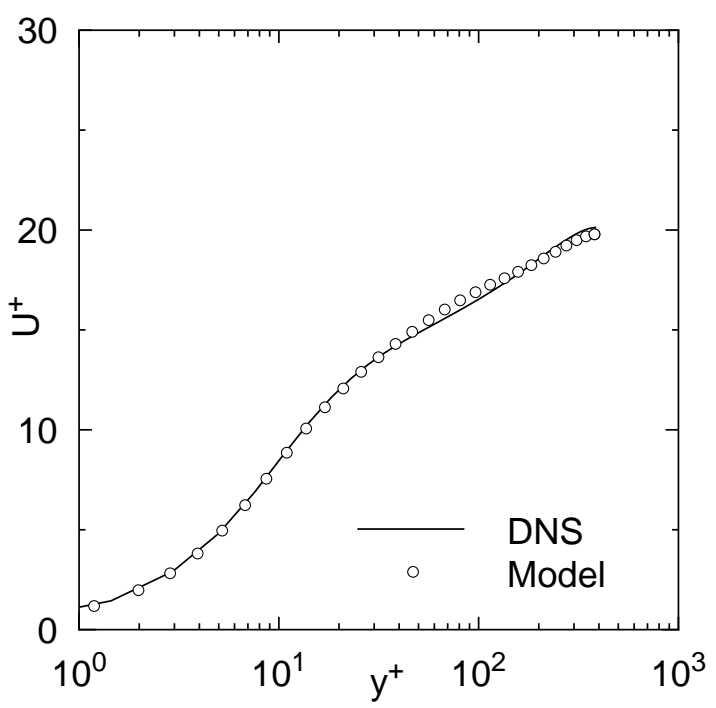

(a)

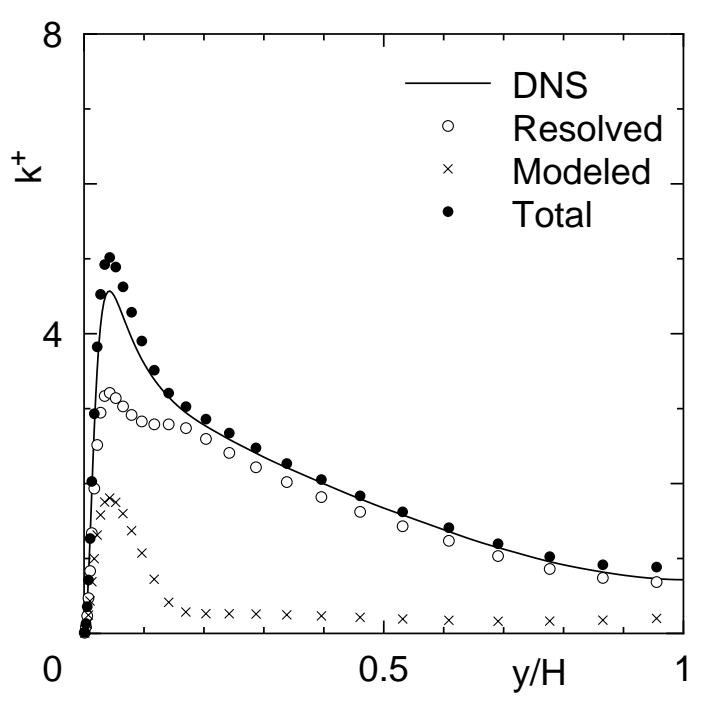

(c)

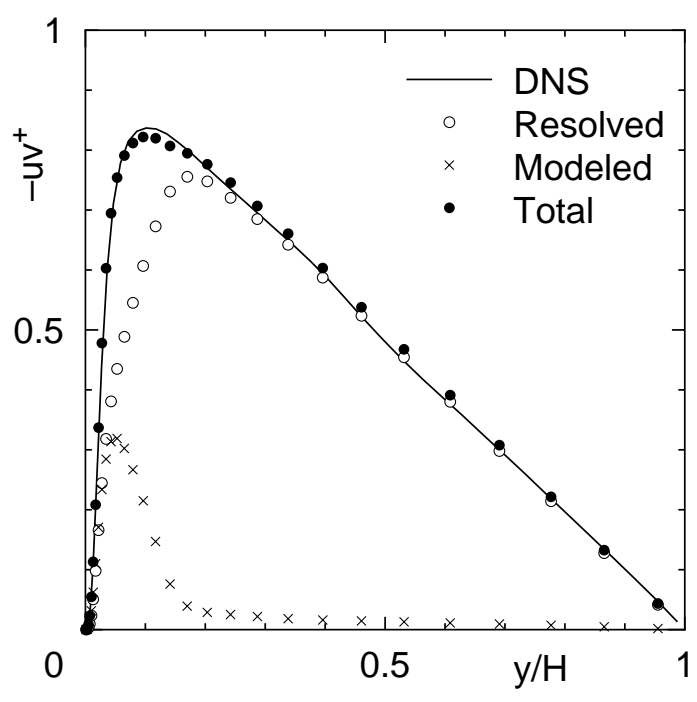

(b)

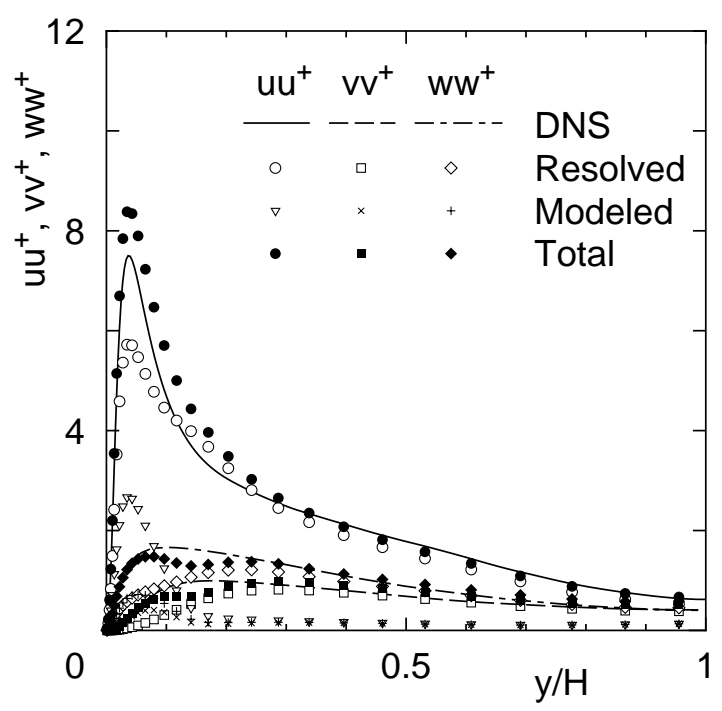

(d)

Figure 6:

$\ll \mathrm{K}$. Abe» 


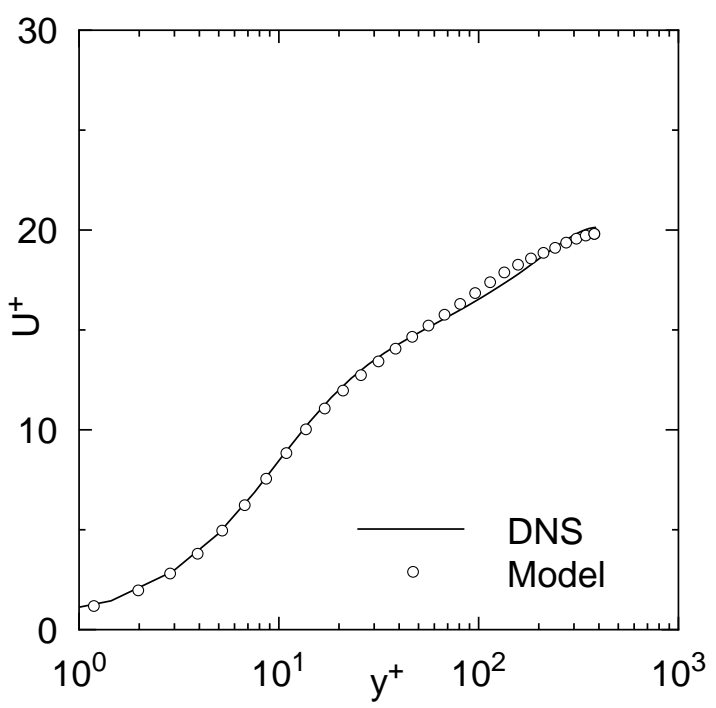

(a)

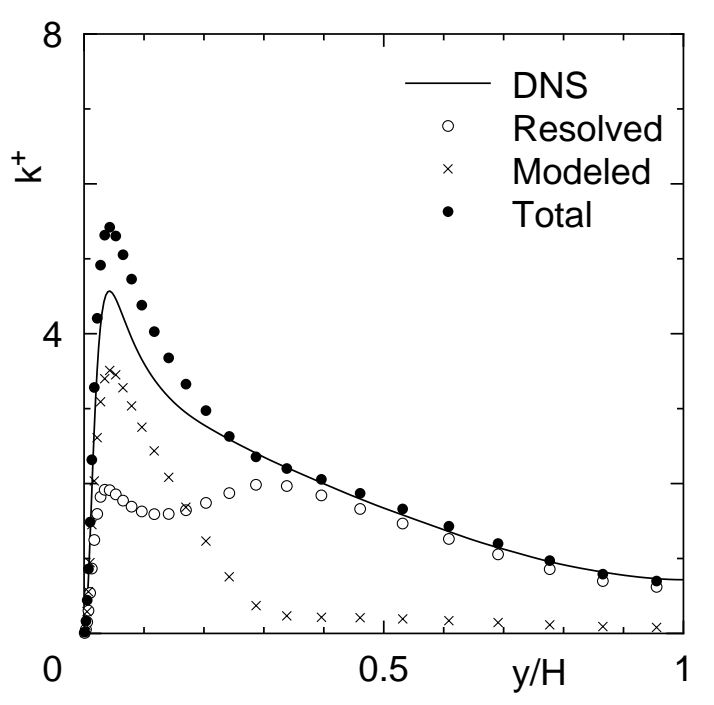

(c)

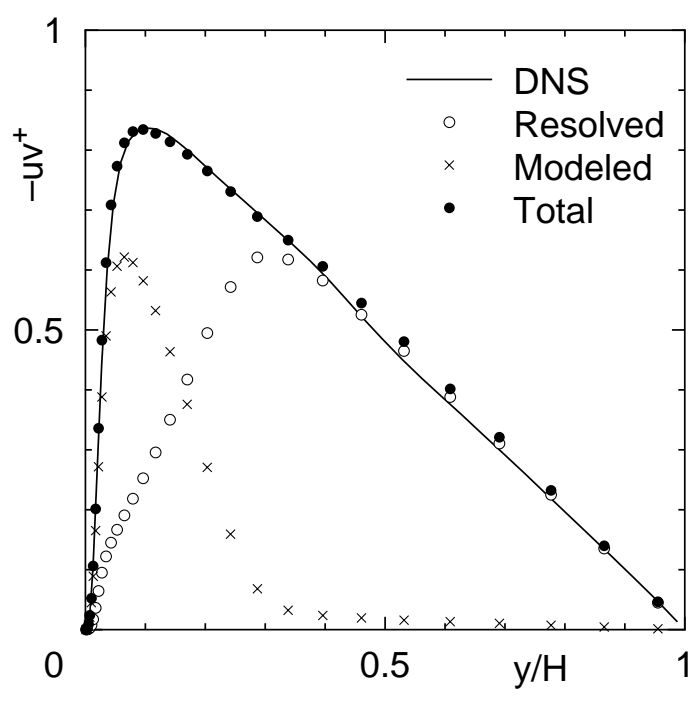

(b)

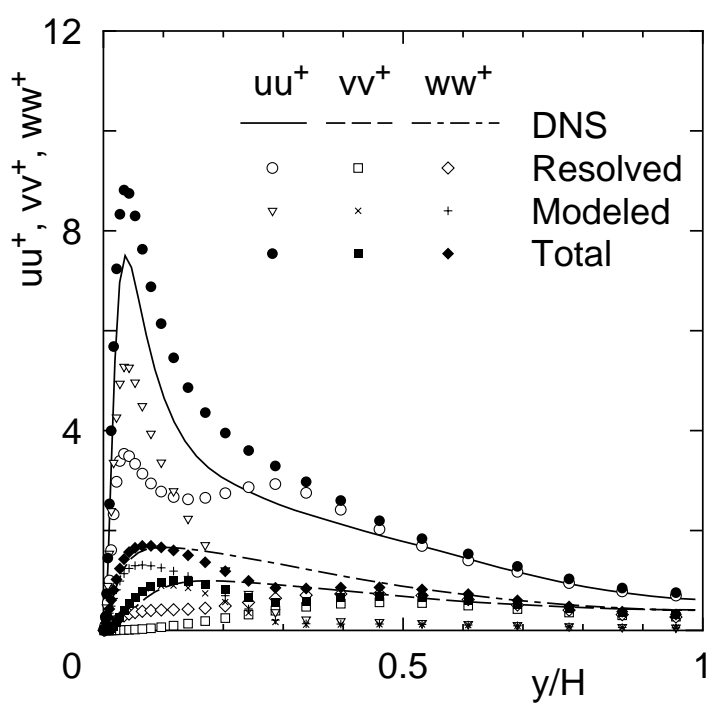

(d)

Figure 7:

$\ll \mathrm{K}$. Abe $\gg$ 


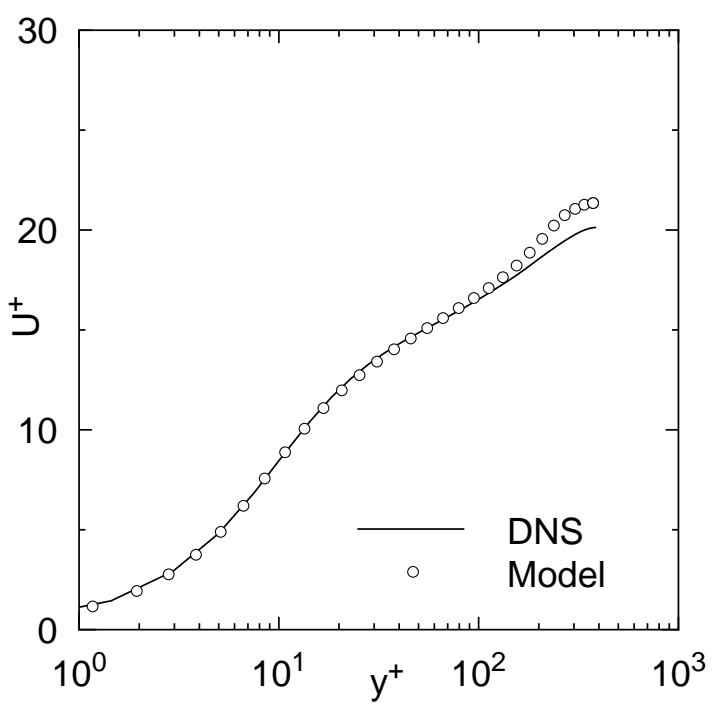

(a)

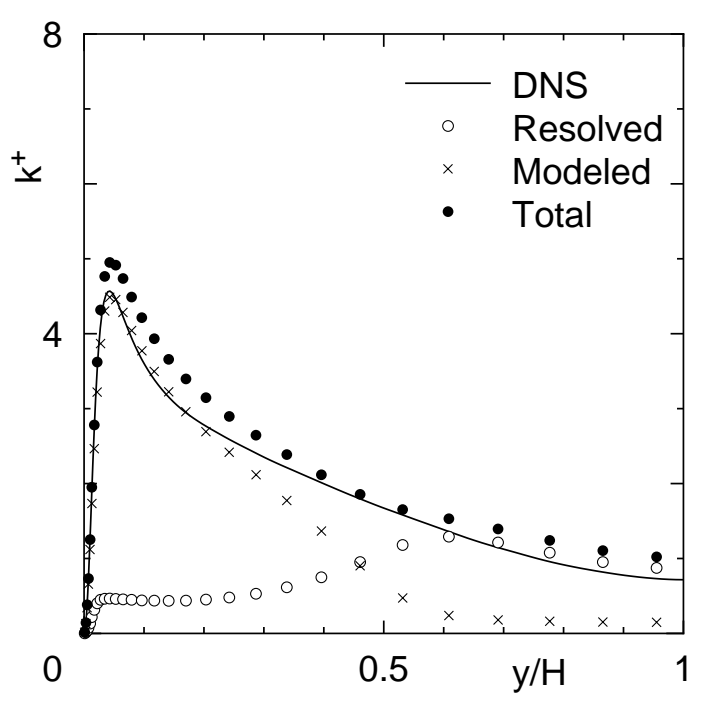

(c)

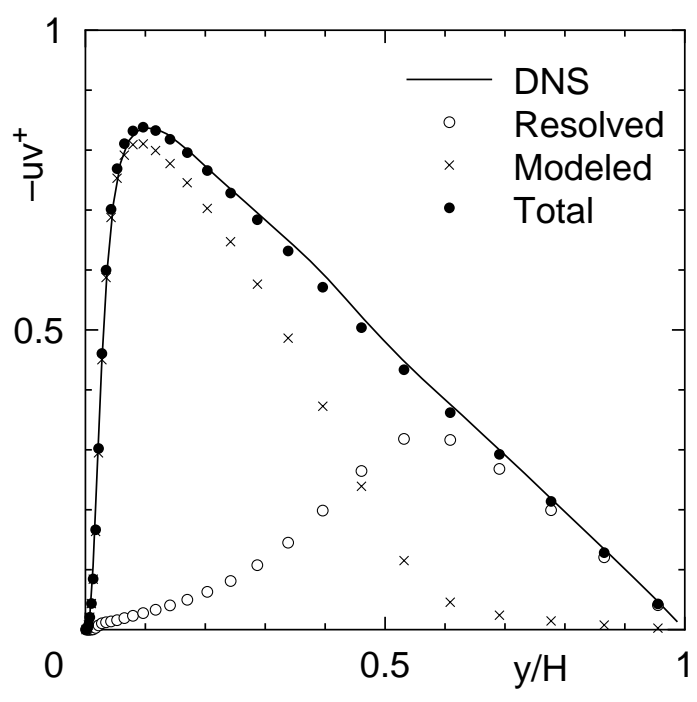

(b)

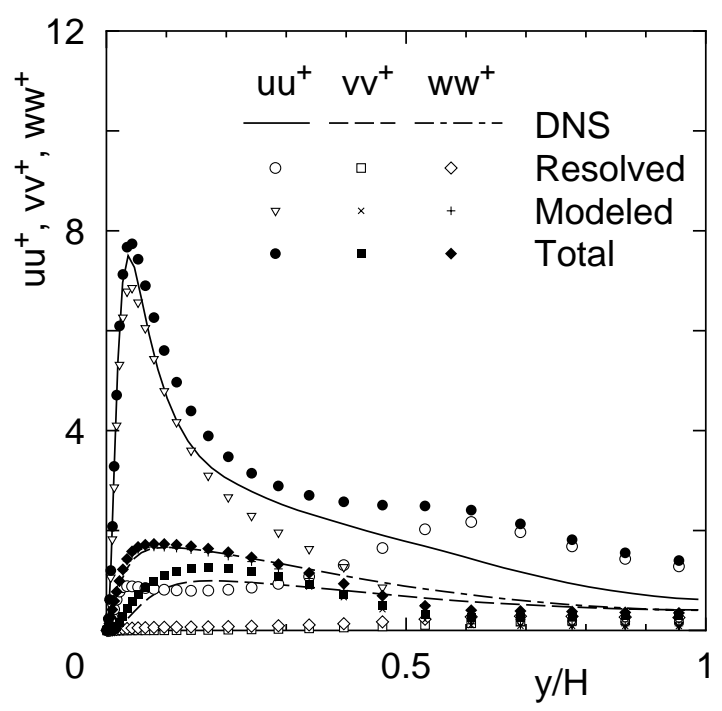

(d)

Figure 8:

$\ll \mathrm{K}$. Abe» 


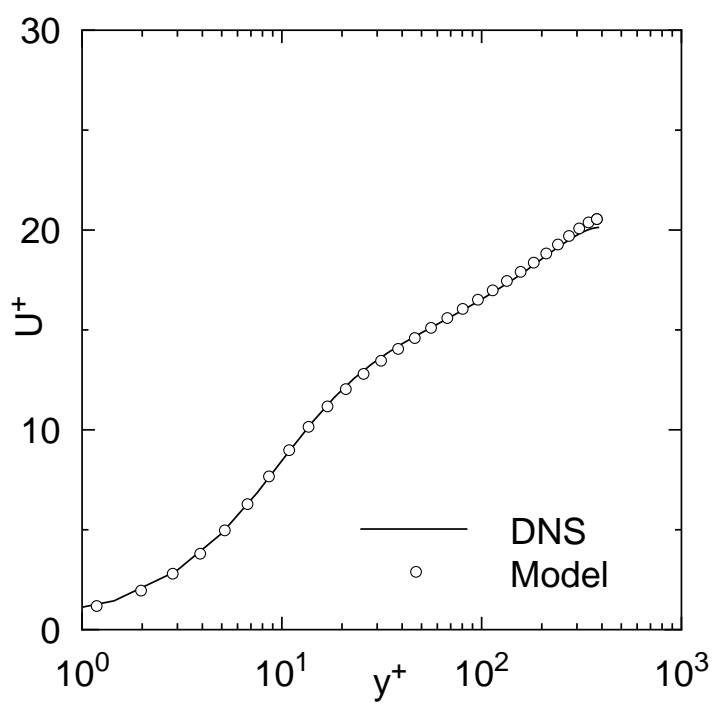

(a)

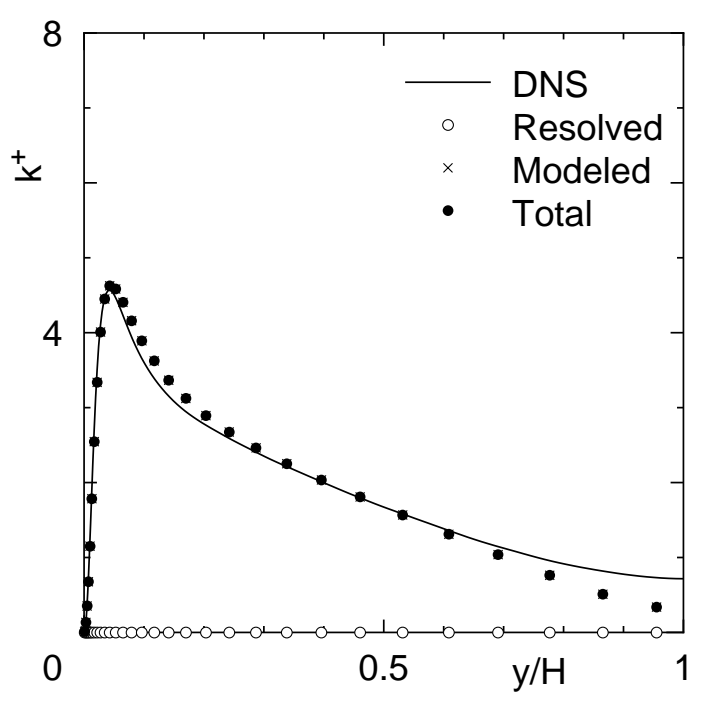

(c)

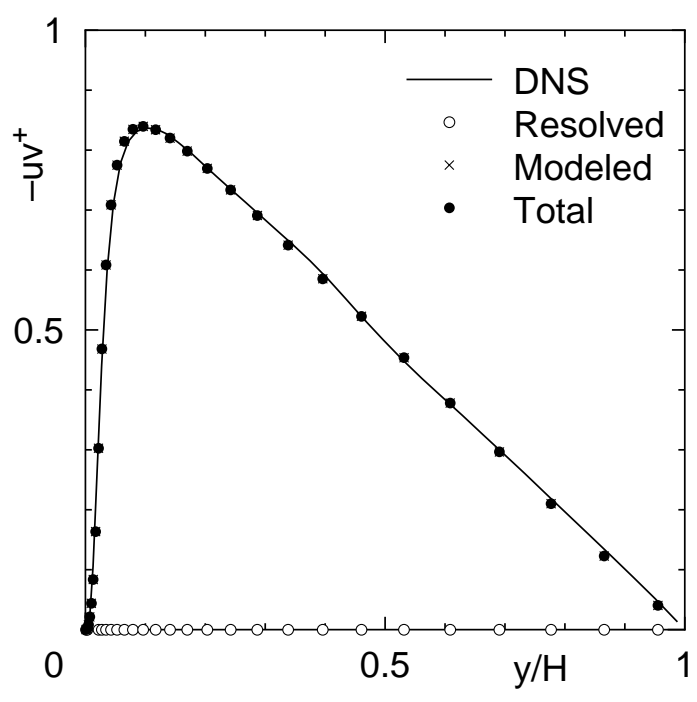

(b)

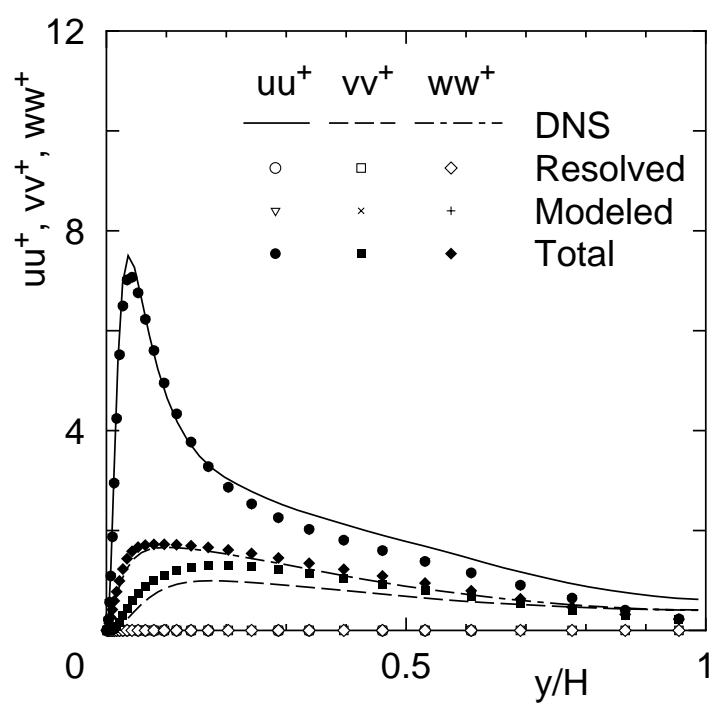

(d)

Figure 9:

$\ll \mathrm{K}$. Abe» 


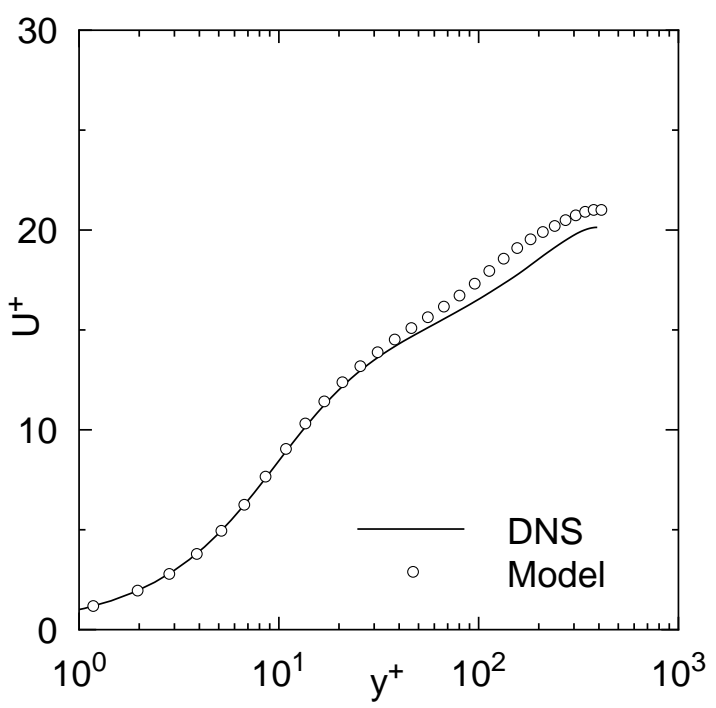

(a)

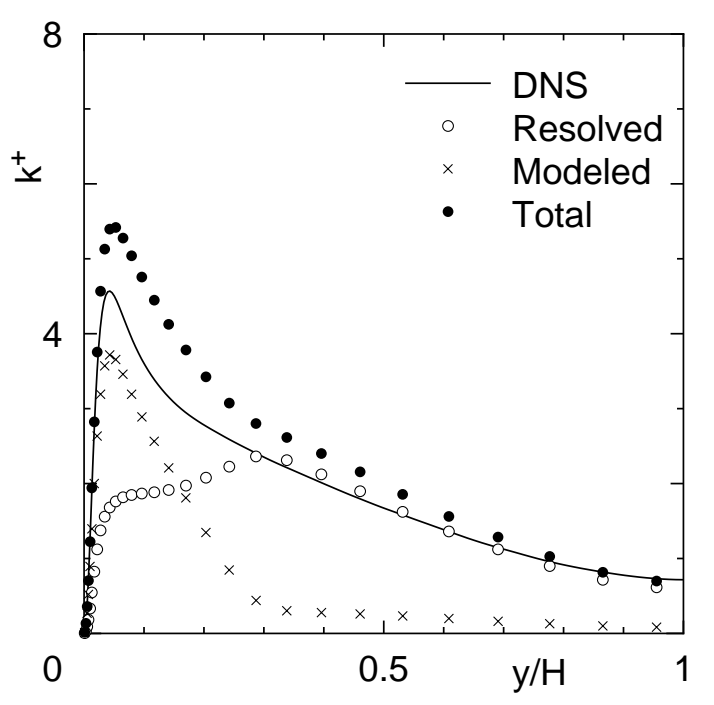

(c)

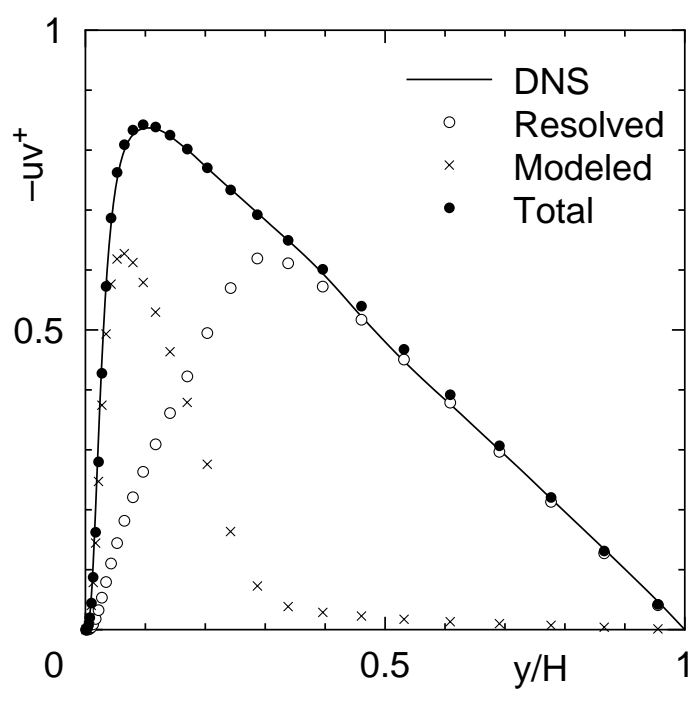

(b)

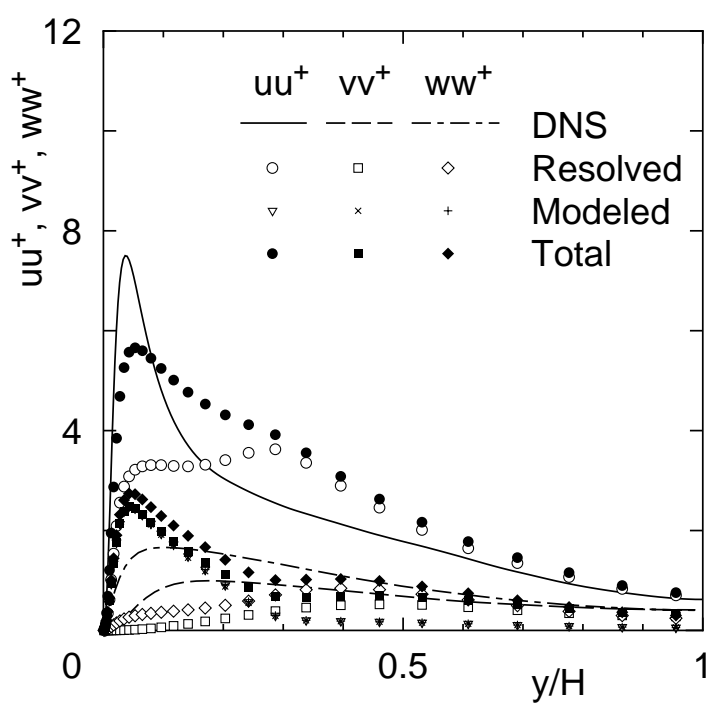

(d)

Figure 10:

$\ll \mathrm{K}$. Abe» 


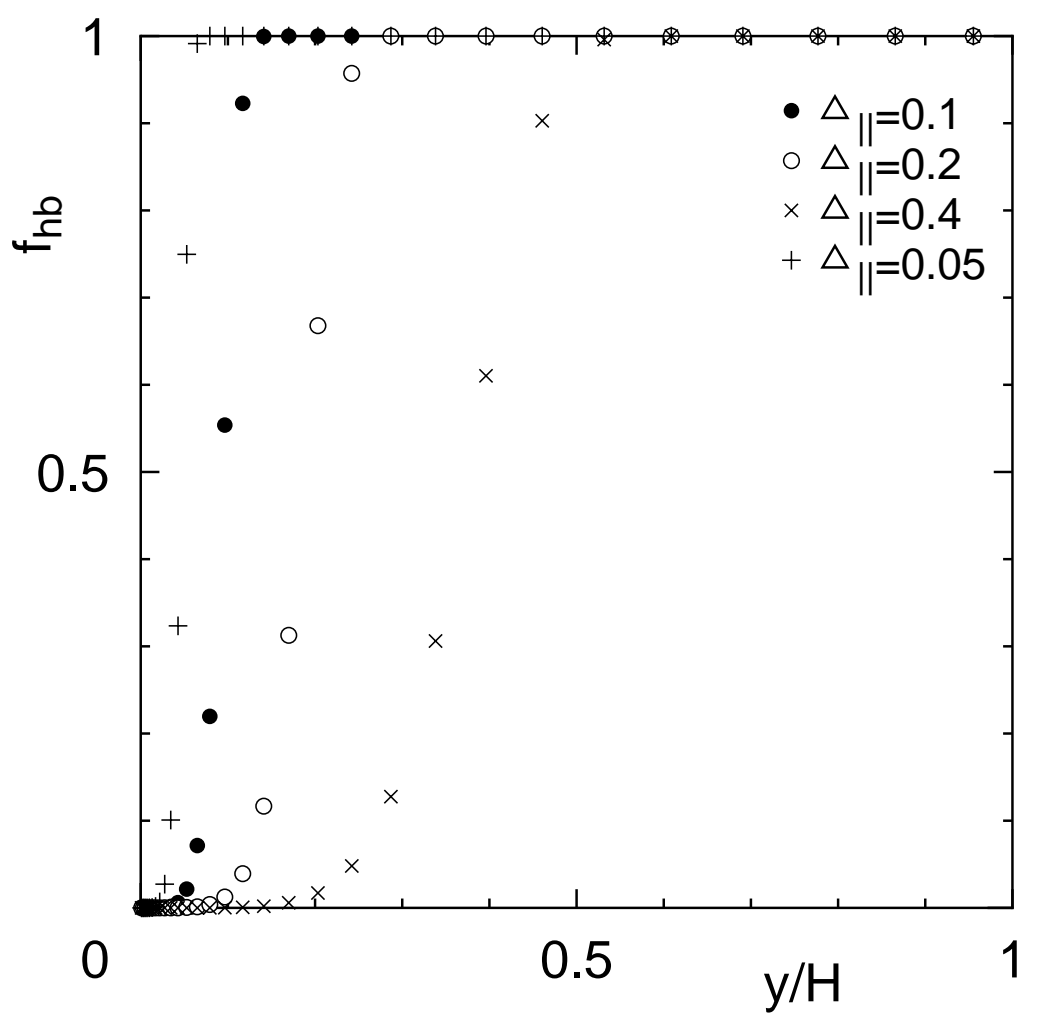

Figure 11: $\quad \ll K$. Abe» 


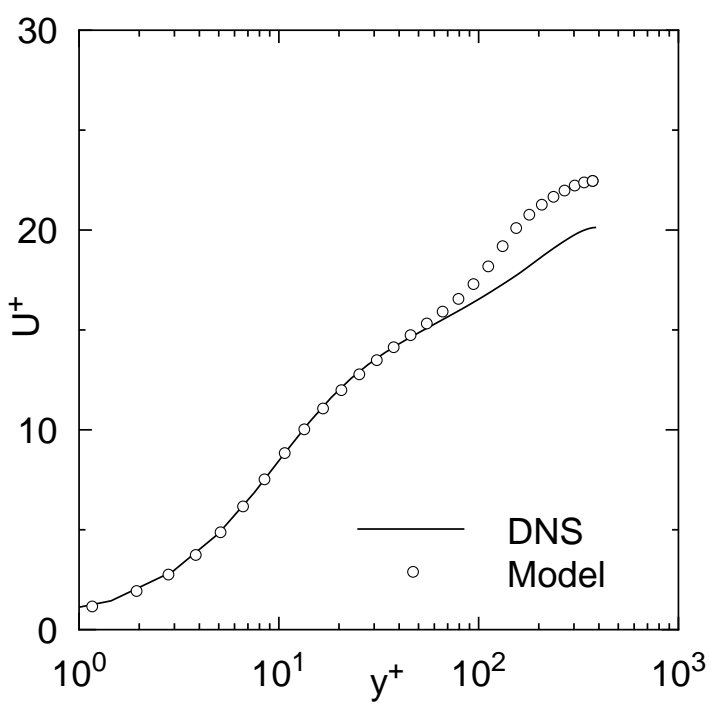

(a)

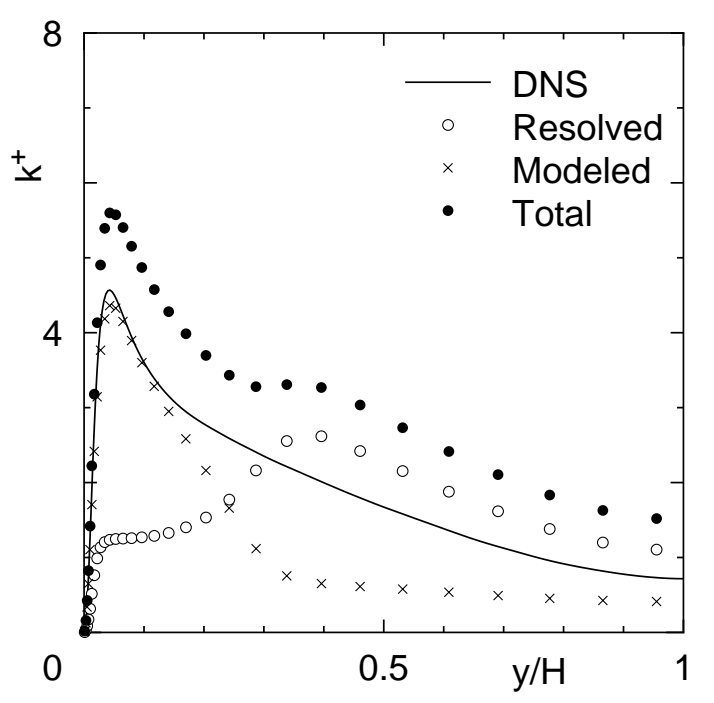

(c)

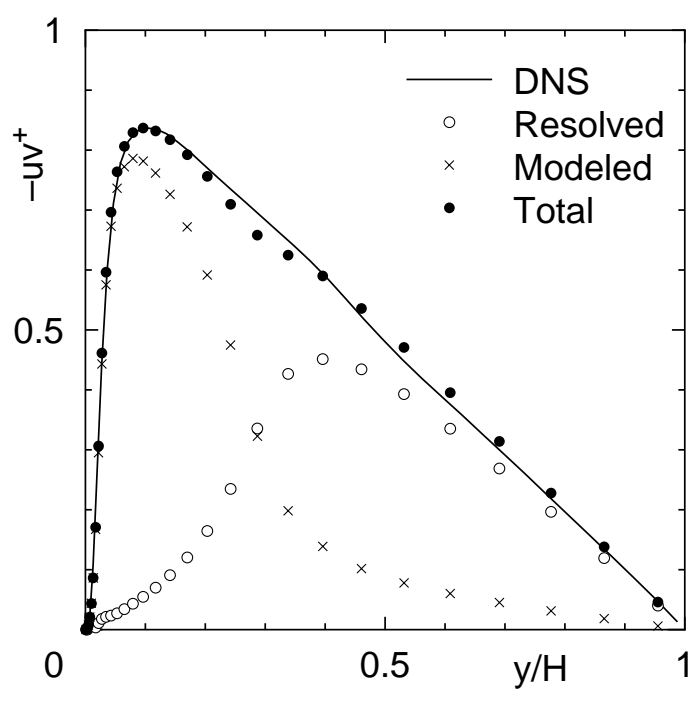

(b)

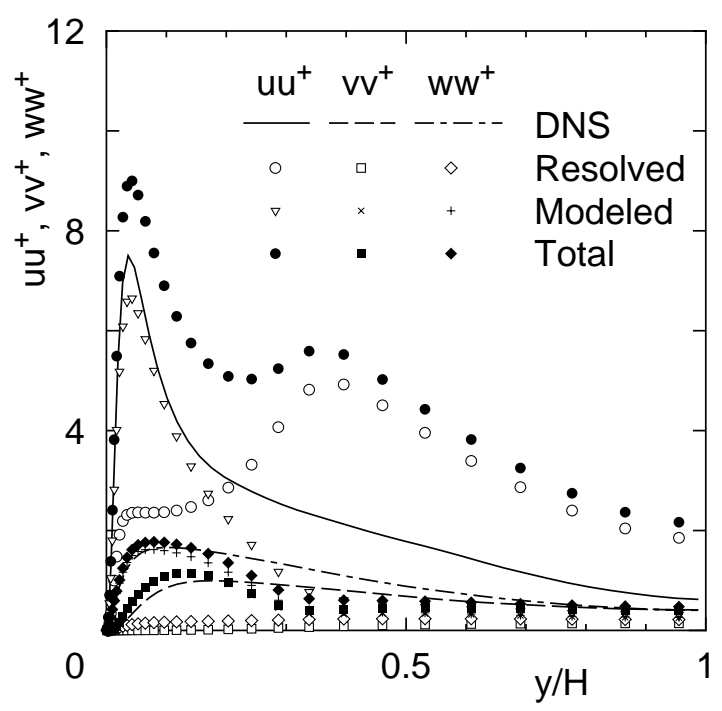

(d)

Figure 12: $\quad \ll \mathrm{K}$. Abe $\gg$ 

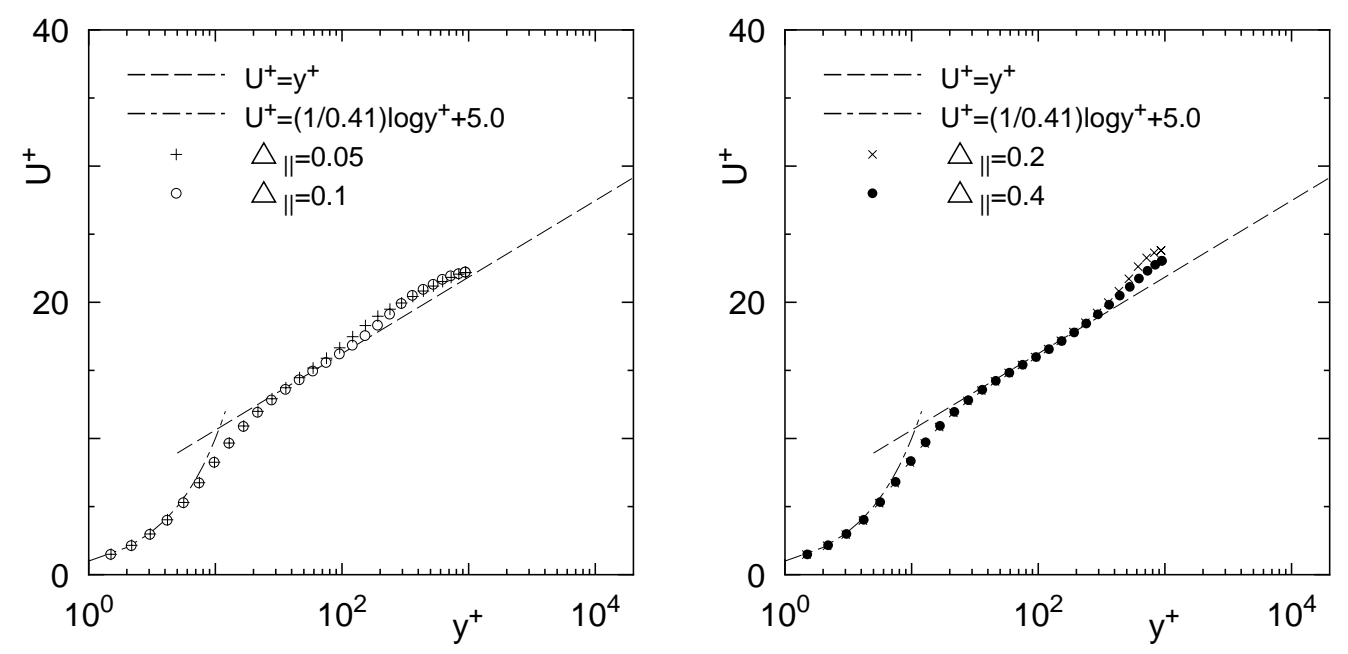

(a)
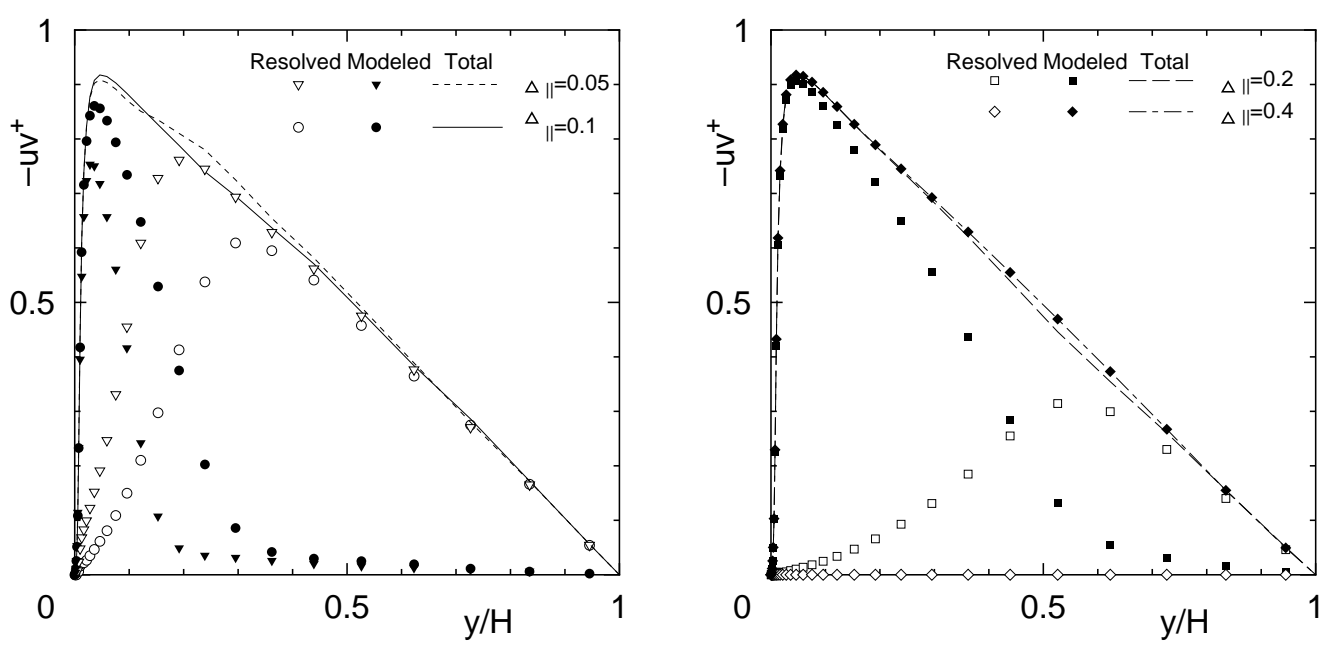

(b)
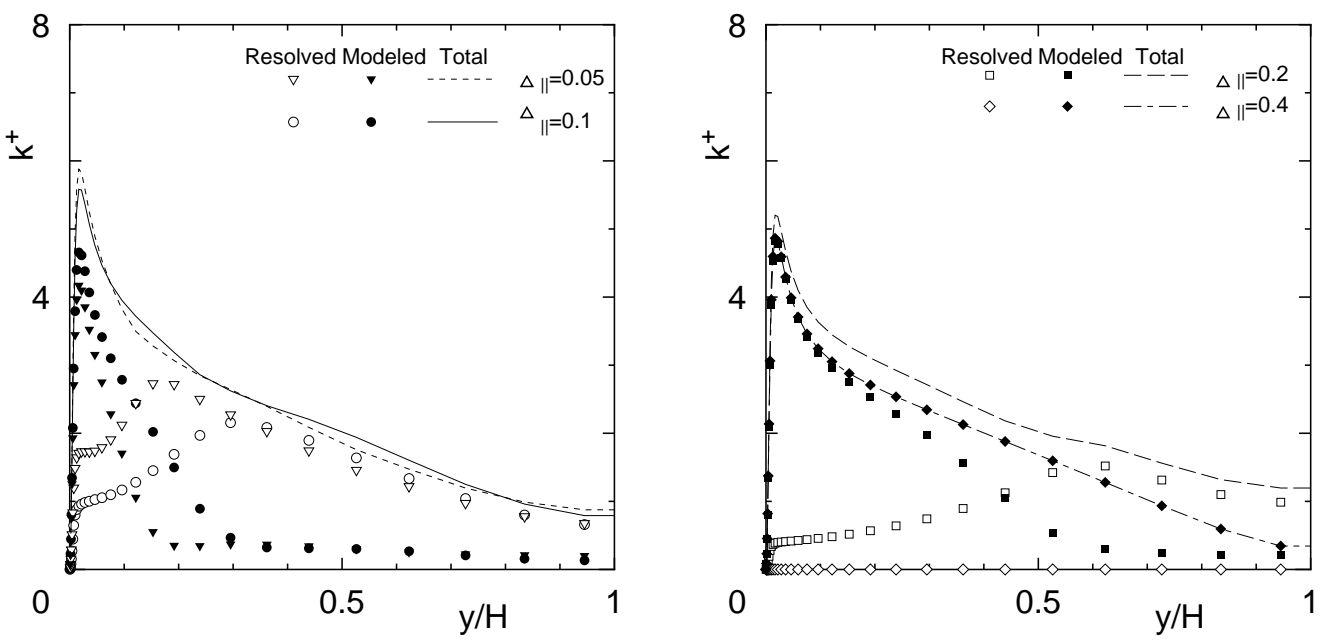

(c)

Figure 13: $\quad \ll$ K. Abe» 

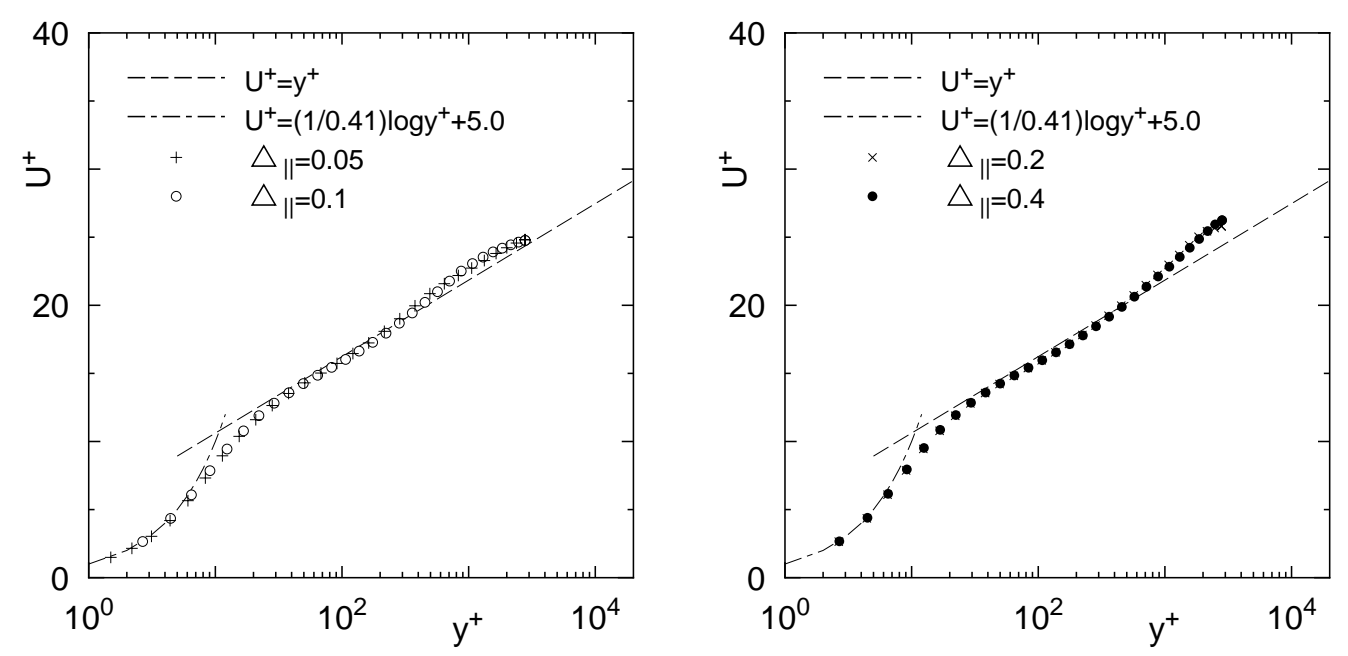

(a)
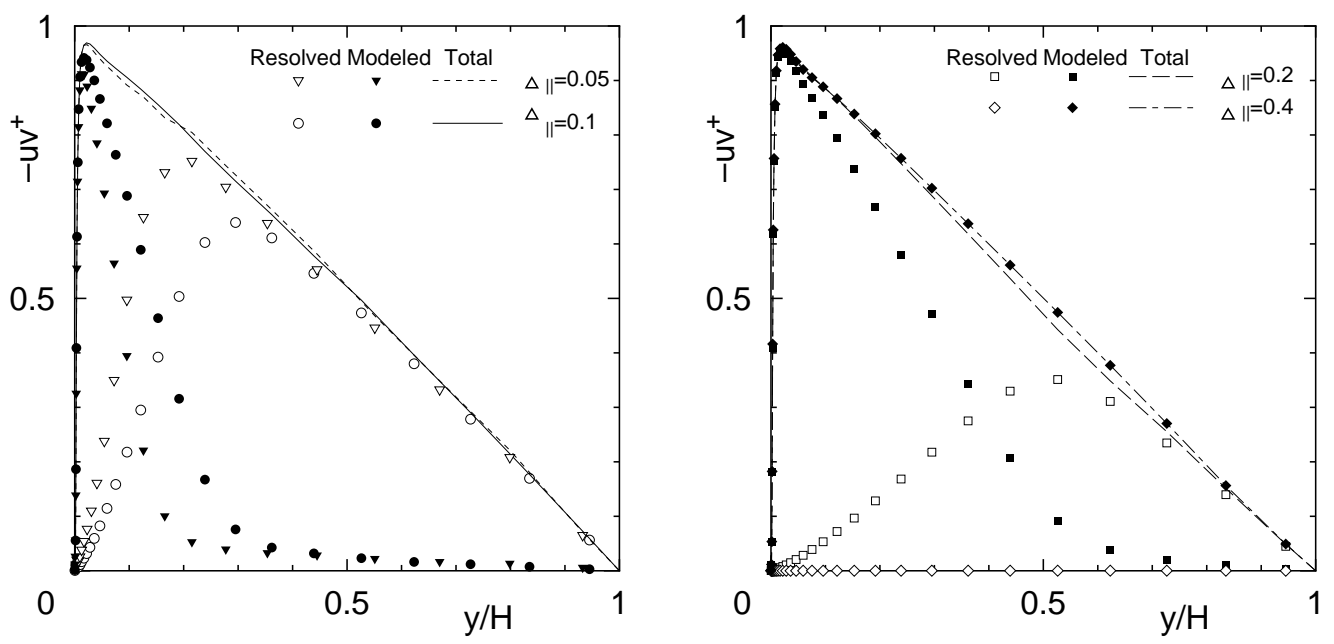

(b)
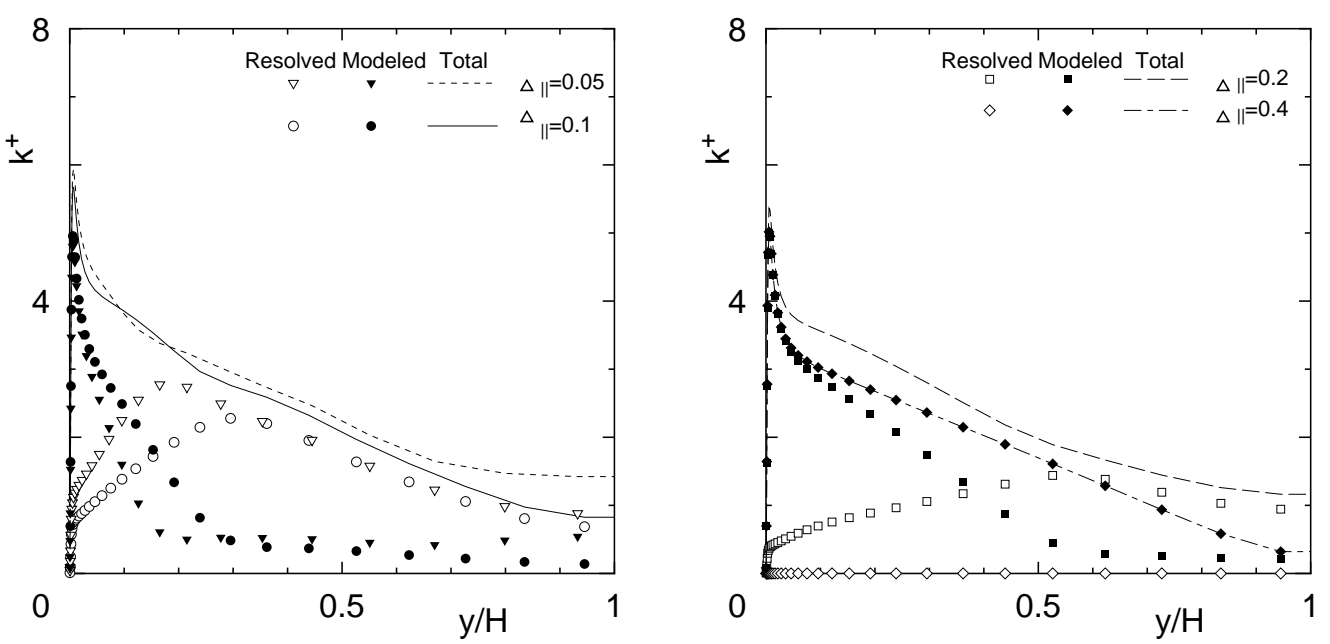

(c)

Figure 14: $\quad \ll K$. Abe» 

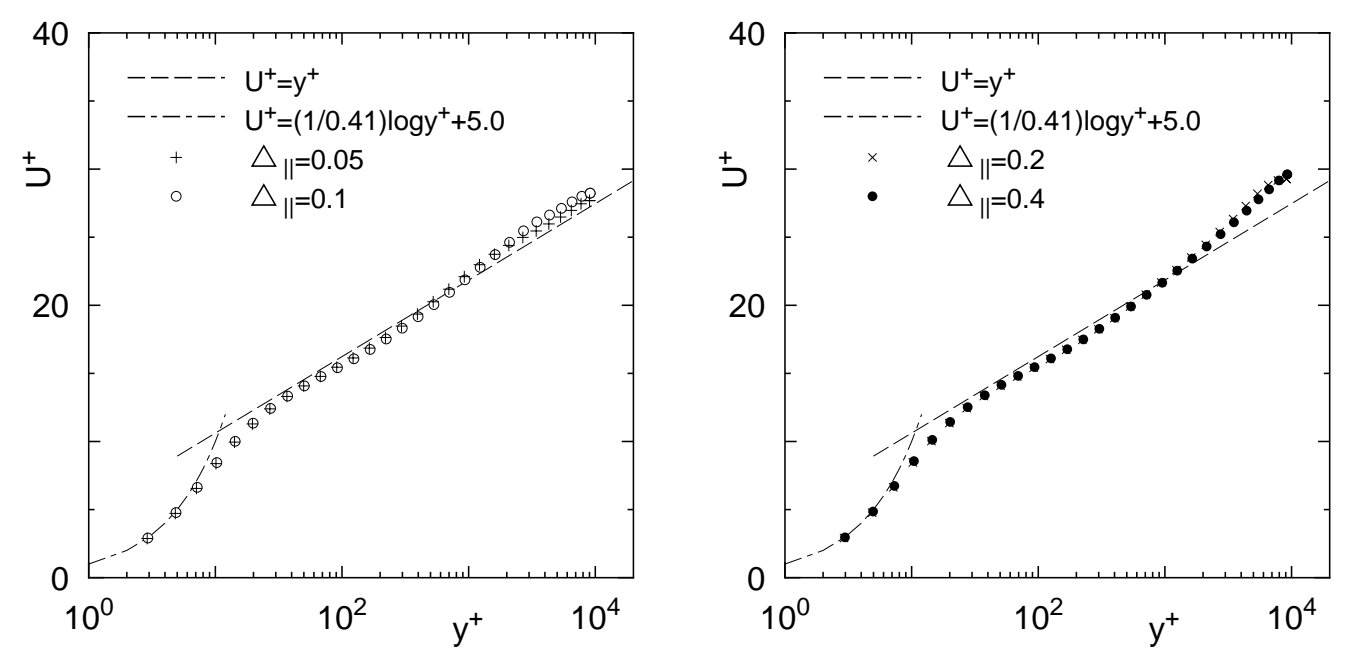

(a)
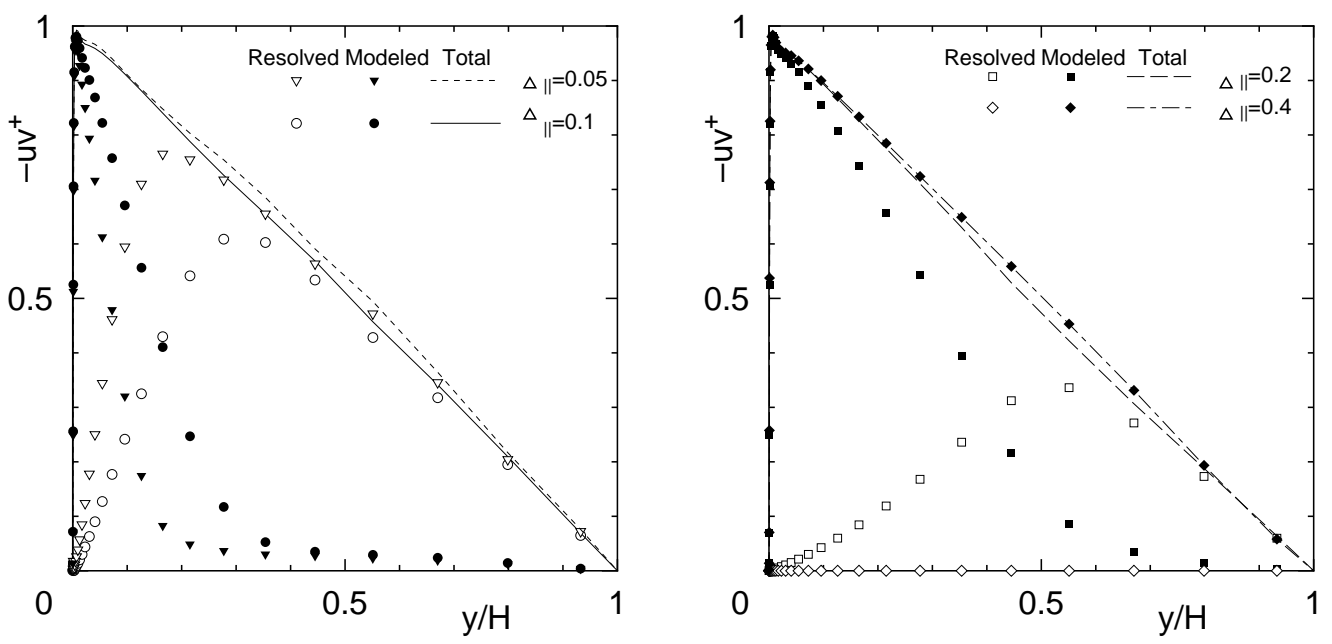

(b)
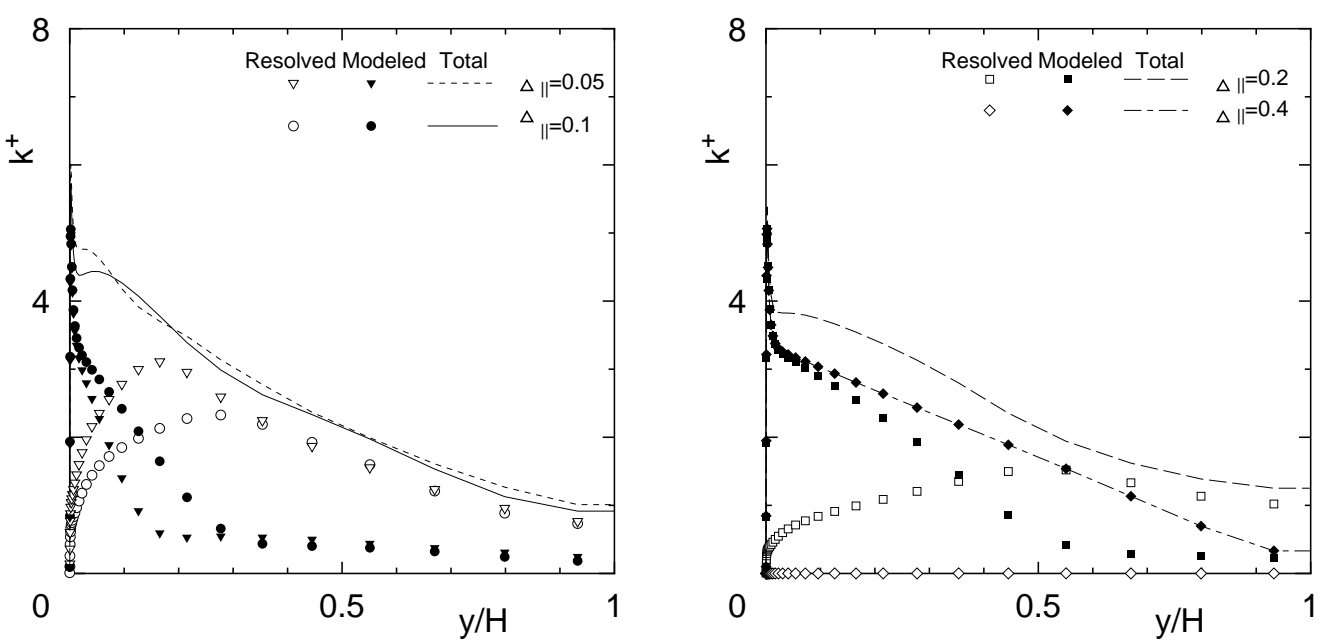

(c)

Figure 15: $\quad \ll K$. Abe» 


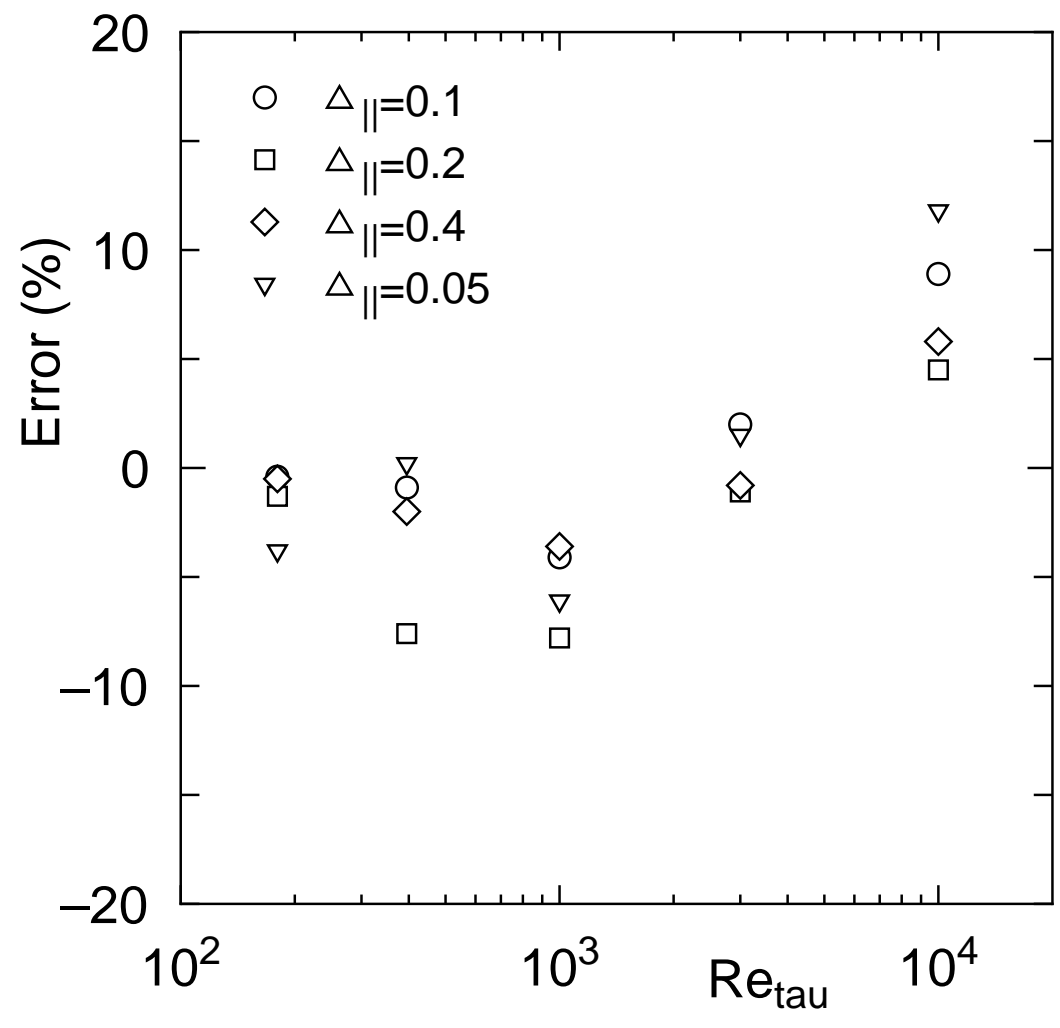

Figure 16: $\quad \ll \mathrm{K}$. Abe» 